
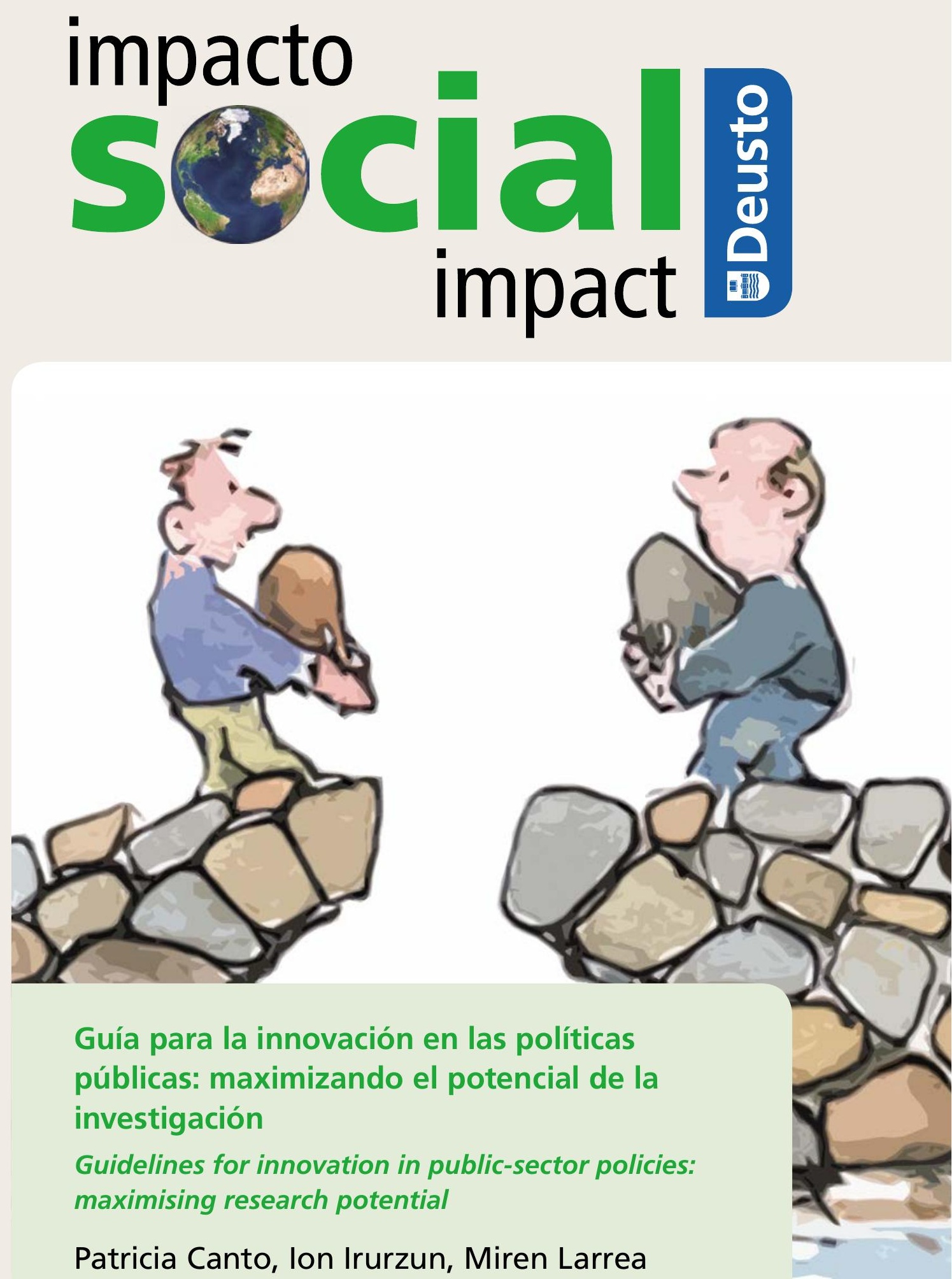

Guía para la innovación en las políticas públicas: maximizando el potencial de la investigación

Guidelines for innovation in public-sector policies: maximising research potential

Patricia Canto, Ion Irurzun, Miren Larrea 



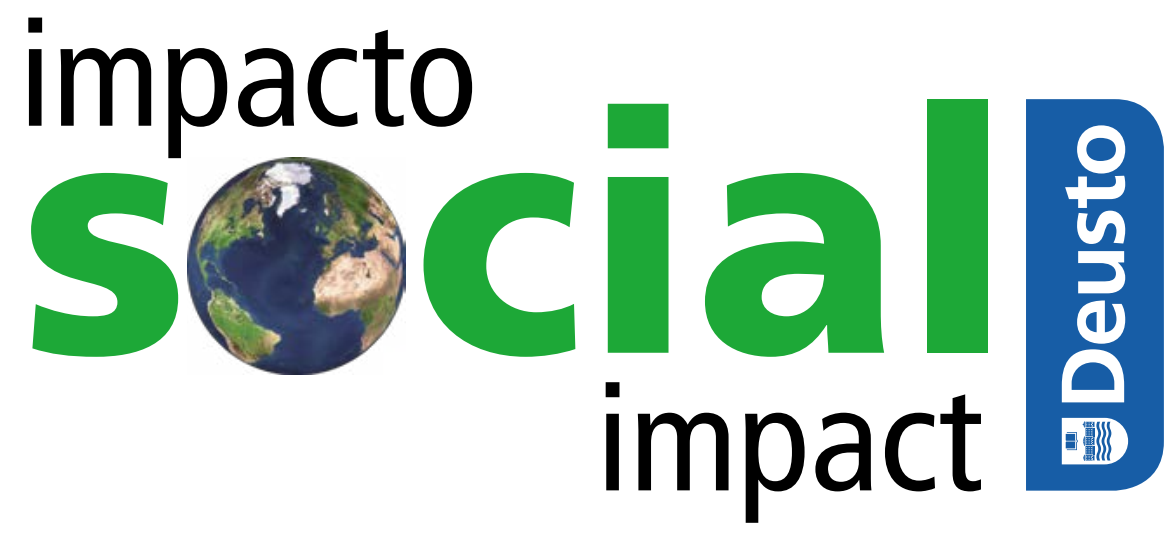


En el marco de la labor llevada a cabo por la Universidad de Deusto (http://www.deusto.es/) en el tema del impacto social de la investigación, anualmente se seleccionan una serie de proyectos de investigación con alto potencial de impacto social, y a partir de ellos, se elaboran y publican los denominados Deusto Social Impact Briefings (DSIB). Son unas monografías breves dirigidas a instituciones sociales, usuarios, policy makers, o empresas que, en lenguaje no académico, responden al objetivo de poner de manifiesto la acción transformadora de la investigación de Deusto, posibilitando que los resultados de la investigación de Deusto sean inteligibles para los agentes sociales y puedan ayudarles a responder a los retos de transformación social a los que se enfrentan, ofreciéndoles buenas prácticas, guías o recomendaciones en la labor que desempeñan.

\section{Frecuencia de publicación y formato}

Deusto Social Impact Briefings se publica electrónicamente y en versión impresa una vez al año. Su primer número se publicó en enero de 2017.

\section{Suscripciones}

Actualmente, no se aplican cargos por la presentación, publicación, acceso en línea y descarga. Pocas copias impresas se ponen a disposición de los colaboradores y socios clave.

\section{Derechos de autor}

Deusto Social Impact Briefings es una publicación de Acceso Abierto de la Universidad de Deusto (España).

Su contenido es gratuito para su acceso total e inmediato, lectura, búsqueda, descarga, distribución y reutilización en cualquier medio o formato sólo para fines no comerciales y en cumplimiento con cualquier legislación de derechos de autor aplicable, sin la previa autorización del editor o el autor; siempre que la obra original sea debidamente citada y cualquier cambio en el original esté claramente indicado. Cualquier otro uso de su contenido en cualquier medio o formato, ahora conocido o desarrollado en el futuro, requiere el permiso previo por escrito del titular de los derechos de autor.
Within the framework of the work carried out by the University of Deusto (http://www.deusto.es/) on the social impact of research, a series of research projects with high potential for social impact are selected annually, and from these, the so-called Deusto Social Impact Briefings (DSIB) are prepared and published as short monographs. They are aimed at social organisations, users, policymakers and businesses. They seek to ensure that research outcomes are intelligible to all these different social actors. They also seek to provide guidelines, best practices and recommendations to support the tasks of meeting the challenges of social transformation that must be faced.

\section{Publication frequency and format}

Deusto Social Impact Briefings is published electronically and in print version once a year. Its first issue appears in January 2017.

\section{Subscriptions}

Currently, no charges for submission, publication, online access, and download are applicable. Few print copies are freely made available for key collaborators and partners.

\section{Copyrights}

Deusto Social Impact Briefings is an Open Access publication of the University of Deusto (Spain).

Copyright for this publication is retained by the Publisher. Any part of its content can be reused in any medium or format only for non-commercial purposes and in compliance with any applicable copyright legislation, without prior permission from the Publisher or the author(s). In any case, proper acknowledgement of the original publication source must be made and any changes to the original work must be clearly indicated. Any other use of its content in any medium or format, now known or developed in the future, requires prior written permission of the copyright holder.

(C) Universidad de Deusto

P.O. box 1 - 48080 Bilbao, España

Publicaciones

Tel.: +34-944139162

E-mail: publicaciones@deusto.es

URL: www.deusto-publicaciones.es

ISBN: 978-84-16982-16-5 (version impresa / printed version)

Deposito Legal/ Legal Deposit: BI-108-2017

Impreso y encuadernado en España / Printed

and bound in Spain 
DEUSTO Social Impact Briefings No. 1 (2016)

Dirección y coordinación editorial

Cristina Iturrioz Landart, Universidad de Deusto, España Antonia Caro González, Universidad de Deusto, España

\section{Comité Cientifico:}

Javier Arellano Yanguas, Director del Centro de Ética Aplicada e investigador principal del equipo «Ética Aplicada a la Realidad Social» (EARS), Universidad de Deusto

Antonia Caro González, Directora de la Oficina de Proyectos Internacionales de Investigación y experta en temas europeos, innovación en gestión de la investigación e impacto social, Universidad de Deusto, España

Laura Teresa Gómez Urquijo, investigadora y Profesora en la Facultad de Derecho e investigadora del equipo Desarrollo Social, economía e Innovación para las Personas (EDISPE), Universidad de Deusto, España

Cristina Iturrioz Landart, Vicerrectora de Investigación y Transferencia e investigadora del equipo de Competitividad Empresarial y Desarrollo Económico (CEDE), Universidad de Deusto, España

Amaia Méndez Zorrilla, Professora en la Facultad de Ingenieria e investigadora del equipo E-vida, Universidad de Deusto, España

José Javier Pardo Izal, Profesor del departamento de Teología e investigador en Teología Bíblica y su influencia cultural, Universidad de Deusto, España.

Rosa María Santibañez Gruber, Profesora en la Facultad de Psicologia y Educación e investigadora principal del equipo Intervención: Calidad de Vida e Inclusión Social, Universidad de Deusto, España

\section{Editors}

Cristina Iturrioz Landart, University of Deusto, Spain Antonia Caro González, University of Deusto, Spain

\section{Scientific Committee:}

Javier Arellano Yanguas, Head of the Centre for Applied Ethics and Main Researcher in the Ethics Applied to Social Reality research team at the University of Deusto, Spain.

Antonia Caro González, Head of the International Research Project Office and expert in European issues, social impact and innovation in research management at the University of Deusto, Spain

Laura Teresa Gómez Urquijo, lecturer at the Faculty of Law and researcher in the Economics, Social Development and Innovation for Persons (EDISPE) team at the University of Deusto, Spain

Cristina Iturrioz Landart, Deputy Rector for Research and Knowledge Transfer and researcher in the Business Competitiveness \& Economic Development (CEDE) team at the University of Deusto, Spain

Amaia Méndez Zorrilla, lecturer at the Faculty of Engineering and researcher in the E-Life team at the University of Deusto, Spain

José Javier Pardo Izal, lecturer at the Department of Theology and researcher working on biblical theology and its cultural influence at the University of Deusto, Spain.

Rosa María Santibañez Gruber, lecturer at the Faculty of Psychology and Education and Main Researcher in the Intervention: Quality of Life and Social Inclusion team at the University of Deusto, Spain 



\section{Prólogo}

Los Deusto Social Impact Briefings (DSIB) son monografías breves que permiten mostrar las capacidades de investigación instaladas en la Universidad de Deusto (http://www.deusto.es/) a través de proyectos de investigación desarrollados por personal investigador de nuestra universidad en las áreas de conocimiento de alta relevancia social. Publicados en papel y online (en castellano e inglés) con una periodicidad anual, se pueden encontrar también en la página web de Deusto Research.

Están dirigidos a entidades sociales, usuarios, policy makers y/o empresas, posibilitando que los resultados de la investigación sean inteligibles para estos diferentes agentes sociales y pretenden, mediante la oferta de buenas prácticas, guías o recomendaciones, apoyarles en la labor que desempeñan para responder a los retos de transformación social a los que se enfrentan.

Dentro del proyecto estratégico Deusto 2018 y en el marco del Plan Director de Impacto Social, el presente briefing fue seleccionado, por su alto potencial de impacto social, a través de una convocatoria lanzada a toda la comunidad investigadora en 2016. Forma parte de un conjunto de cinco briefings resultantes de la mencionada convocatoria y que conforman el primer número de los DSIB.

El Guía para la innovación en las políticas públicas: maximizando el potencial de la investigación se plantea, en base a una experiencia de colaboración investigación-acción, una metodología de trabajo conjunto entre personal investigador y agentes públicos para el desarrollo de políticas regionales.

Deseamos disfruten de su lectura tanto como nosotros lo hemos hecho durante la preparación de la publicación; ha sido especialmente gratificante comprobar el grado de implicación y colaboración del personal investigador académico y de los 'stakeholders' en los proyectos.

Esperamos esta publicación cumpla el objetivo para el que ha sido creado, que no es otro que el de hacer la ciencia más accesible, útil y cercana a profesionales, responsables de políticas públicas y agentes sociales que trabajáis en los temas. Agradecemos vuestro interés y estamos abiertos a nuevas iniciativas, así como a comentarios y sugerencias que nos queráis hacer llegar.

Cristina Iturrioz Landart, Dirección DSIB Antonia Caro González, Dirección DSIB

Diciembre de 2016 


\section{Foreword}

The Deusto Social Impact Briefings (DSIB) are brief monographic publications that highlight the research capabilities based at the University of Deusto (http:// www.deusto.es/) through projects conducted by researchers at our University in knowledge areas with a high level of social relevance. They are to be published yearly in hard copy and on-line (in both Spanish and English) and can also be found on the website of Deusto Research.

They are aimed at social organisations, users, policy-makers and businesses, and seek to ensure that research outcomes are intelligible to all these different social actors. They also seek to provide guidelines, best practices and recommendations to support the tasks of meeting the challenges of social transformation that must be faced.

As part of the Deusto 2018 strategic project under the Master Plan for Social Impact, this briefing was selected for its high potential social impact, following a call sent out to the whole research community in 2016. It is one of five briefings selected from that call for inclusion in the first issue of the DISB.

Guidelines for Innovation in Public-Sector Policies: Maximising Research Potential presents an experience of action research setting out a method for joint work between researchers and public sector for the development of regional policies.

We hope that you will enjoy reading its findings and conclusions as much as we enjoyed preparing them for publication. It has been particularly gratifying to observe the high level of engagement and cooperation on the part of academic researchers and the stakeholders involved in the project.

We also hope that this publication will meet the goals for which it was created, i.e. to make science more accessible, useful and readily available to specialists, heads of public sector policy and social actors working in the relevant fields. Thank you for your interest; we are open to new initiatives and would welcome any comments and suggestions that you may care to make.

Cristina Iturrioz Landart, Editor Antonia Caro González, Editor

December 2016 


\section{Guía para la innovación en las políticas públicas: maximizando el potencial de la investigación}

Patricia Canto, Ion Irurzun, Miren Larrea

1. Introducción: ¿Por qué una guía para la innovación en las políticas públicas a través de la investigación? ................ 10

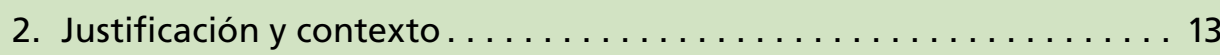

3. Guía para la innovación en políticas de desarrollo territorial ...... 14 FASE 0: Iniciar el proceso: el reto de encontrar un modelo para la colaboración entre policy makers e investigadores. . ......... 15 FASE 1: Creación de las condiciones básicas para el proceso de innovación en políticas ............................. 19 FASE 2: Definición de una agenda compartida ............ 21 FASE 3: Construcción de un nuevo modelo de relación ........... 26 FASE 4: La innovación en las políticas como resultado del proceso. . . 27

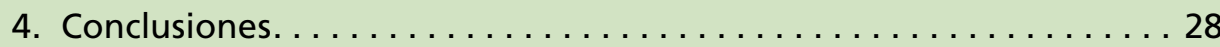

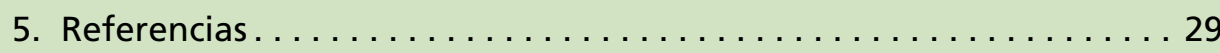

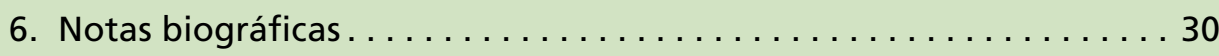

Anexo 1. Publicaciones académicas derivadas del proyecto Gipuzkoa Sarean .................................. 31 



\title{
Guía para la innovación en las políticas públicas: maximizando el potencial de la investigación
}

\author{
Patricia Canto, Ion Irurzun, Miren Larrea
}

doi: http://dx.doi.org/10.18543/dsib-1(2016)-pp147-169.pdf

\section{Resumen}

El documento ofrece una guía para la innovación en las políticas públicas a través de procesos de investigación acción. Plantea a los policy makers que estableciendo este método de trabajo con un equipo de investigación pueden generar espacios en que se tome conciencia de los problemas existentes en los procesos de desarrollo de políticas, se generen nuevos marcos y conceptos para pensar dichos problemas de maneras distintas y se faciliten procesos de decisión que se van implementando de forma incremental, en la medida en que se va aprendiendo.

\section{Palabras clave:}

innovación, políticas, investigación acción, diálogo, confianza

\section{Abstract}

This document sets out guidelines for innovation in public-sector policies through action research processes. It seeks to convey to policy makers the idea that by implementing this working method with research teams they can generate spaces for understanding the problems that exist in policy development processes. It can also serve to generate new frameworks and concepts for thinking about those problems differently, and may thus facilitate decision-making processes that can be implemented incrementally as learning progresses.

\section{Keywords:}

Innovation, policies, action research, dialogue, trust 


\section{Introducción: ¿Por qué una guía para la innovación en las políticas públicas a través de la investigación?}

En el ámbito de las políticas regionales se ha empezado a reconocer la importancia de incorporar a nuevos actores a espacios deliberativos, e incluso de decisión de las políticas. Sin embargo, los procesos que se han llevado a cabo en la práctica plantean un grado de dificultad que hace que muchas veces se estanquen. ¿Cuáles son esas dificultades y cómo pueden superarse?

Esta guía se basa en los aprendizajes que se deprenden de un proyecto de investigación acción denominado "Gipuzkoa Sarean», desarrollado entre 2009 y 2016. Pretende ayudar a superar dicho estancamiento y contribuir a llevar a la práctica marcos conceptuales que, si bien a nivel teórico gozan de un amplio reconocimiento, han mostrado dificultades a la hora de su implementación. Tal es el caso de los sistemas regionales de innovación, los modelos de gobernanza colaborativa o las estrategias de especialización inteligente conocidas como RIS3'.

Uno de los factores que ha contribuido a la obtención de resultados por parte de Gipuzkoa Sarean en términos de innovación en la gobernanza y en las políticas ha sido su continuidad a lo largo de diferentes legislaturas. Dicha continuidad ha permitido generar cambios estructurales que en el momento de escribir esta guía se están institucionalizando. Ello ha requerido el compromiso tanto de distintos cargos políticos como de los cuadros técnicos de la administración pública. Para referirnos al conjunto de políticos electos y cuadros técnicos a partir de este momento utilizaremos el término policy makers. El término en inglés se ha mantenido al no encontrar un término en castellano que agrupara a estos dos colectivos.

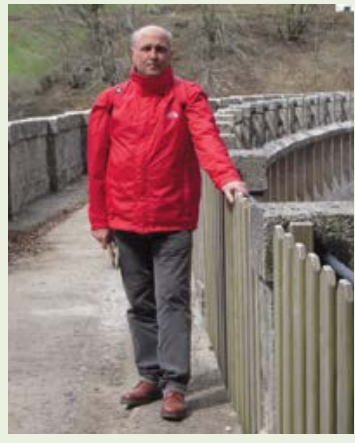

«Cuando el gobierno que inició Gipuzkoa Sarean dejó la diputación nos quedamos los responsables técnicos y los actores académicos únicamente, y todos pensábamos que era un proyecto singular y esperanzador el que teníamos entre manos. De alguna manera nos sentíamos con la responsabilidad del corredor que tienen que sostener el testigo para que no se caiga».

Ander Arzelus, miembro del equipo del Gabinete del Diputado General y participante en Gipuzkoa Sarean desde 2009.

\footnotetext{
1 Para un análisis sobre las dificultades en la implementación de las Estrategias de Especialización Inteligente (RIS3), véase Miren Larrea y Miren Estensoro "Overcoming policy making problems in smart specialization strategies: engaging subregional governments». European Planning Studies, vol. 24 (2016): 1319-1335.
} 


\section{El modelo en que se basa esta guía ha sido co-generado entre investigadores y policy makers}

El eje central de la guía es el modelo Gipuzkoa Sarean para la innovación en las políticas, modelo que se ha construido a través de la investigación acción. Esta metodología, implica un diálogo continuado entre investigadores y policy makers, por lo que el modelo no es resultado exclusivamente de la reflexión académica por parte de los investigadores ni de la práctica de los policy makers. Es un modelo co-generado, es decir, fruto de la combinación de los distintos tipos de conocimiento que aportan tanto los investigadores como los policy makers.

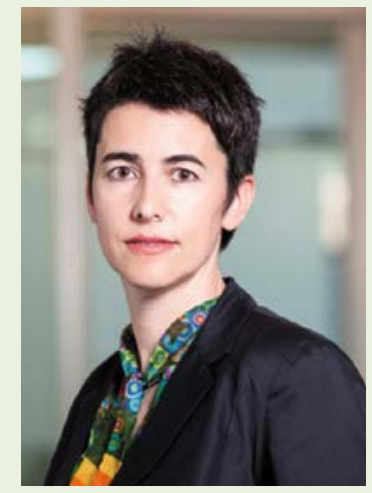

«En 2008 Orkestra hizo una apuesta fuerte por desarrollar la investigación acción. Esta aproximación nos ha dotado de una herramienta muy potente para trabajar con policy makers no «para» la práctica, sino «en» la práctica. Gipuzkoa Sarean ha sido un laboratorio muy importante para desarrollar nuestra propia aproximación a la investigación acción».

Miren Larrea, investigadora de Orkestra, participante en Gipuzkoa Sarean desde 2009 y coordinadora académica desde 2010.

Gipuzkoa Sarean es un proceso de investigación acción orientado a crear y consolidar un modelo de gobernanza basado en la participación. Su objetivo es lograr una mayor eficiencia en el desarrollo de políticas y contribuir a construir una sociedad más democrática. Estos objetivos, y su realización a través del proceso de investigación, han sido compartidos con investigadores internacionales que han validado el proceso.

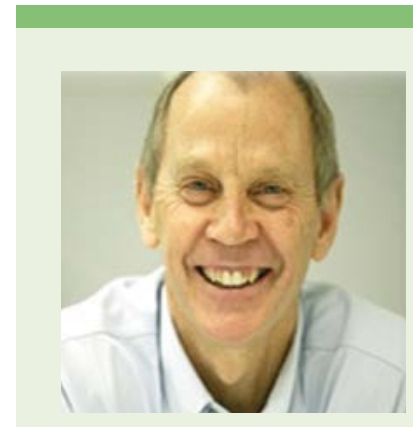

«Lo que se está haciendo aquí es admirable, y hasta donde tengo conocimiento, excepcional. He vivido en muchos sitios y viajado mucho, y nunca he visto ningún gobierno que adoptase la metodología de investigación acción como medio para la transformación. De verdad espero que el proceso tenga éxito y que se constituya como un modelo a seguir».

Ken Dovey, Universidad Tecnológica de Australia. 
El cambio se manifiesta en el comportamiento de los actores, que van configurando un hábito que en el largo plazo se convierte en una nueva cultura
El proyecto de Gipuzkoa Sarean ha estado orientado a la innovación en las políticas, enfatizando para ello la construcción previa de una nueva gobernanza que permita la participación de los actores del territorio. La guía se ha estructurado en torno a las cuatro etapas que esta metodología plantea para innovar en este tipo de procesos.

La consolidación de la nueva gobernanza es el impacto principal del proceso de investigación acción de cara a la innovación en políticas, más allá de los hitos puntuales, consensos parciales y acontecimientos eventuales. Este es un impacto visible tan solo en el largo plazo, que, no obstante, necesita ser legitimado por otros impactos en el corto plazo. Los impactos en el corto plazo en Gipuzkoa Sarean se han materializado en los decretos que se enlistan a continuación y que han incidido en las políticas de desarrollo territorial de Gipuzkoa:

- decreto para estimular programas locales que promuevan el empleo;

- decreto para fomentar el análisis de la cadena de valor de la energía, nuevo esquema de financiación para las micro empresas que invierten en la eficiencia energética;

- acuerdo de internacionalización entre la Diputación Foral de Gipuzkoa, la Cámara de Gipuzkoa y Garapen;

- Organización de eventos en cada comarca para discutir el desarrollo socio económico y construir puentes con otros actores de la comarca.

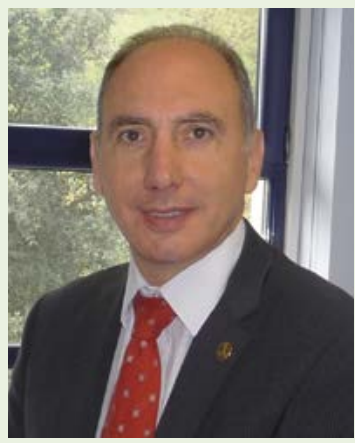

«La clave en el marco de la energía, es orientar las acciones de la comarca, criterios y experiencias a compartir y la importancia de la pedagogía de la energía (tanto para la generación de energía como la eficiencia energética). La participación es importante y hay muchos ejemplos de cómo extenderla, y también diferentes experiencias en los modos de participación».

Juan Angel Balbas, director de la Agencia Comarcal Debabarrena. 


\section{Justificación y contexto}

En el período 2009-2016 en Gipuzkoa Sarean se ha producido un aprendizaje significativo por parte de los policy makers e investigadores participantes sobre cómo abordar la innovación en políticas de desarrollo territorial, sobre todo al abrir estas políticas a la participación de actores del territorio. Este aprendizaje ha

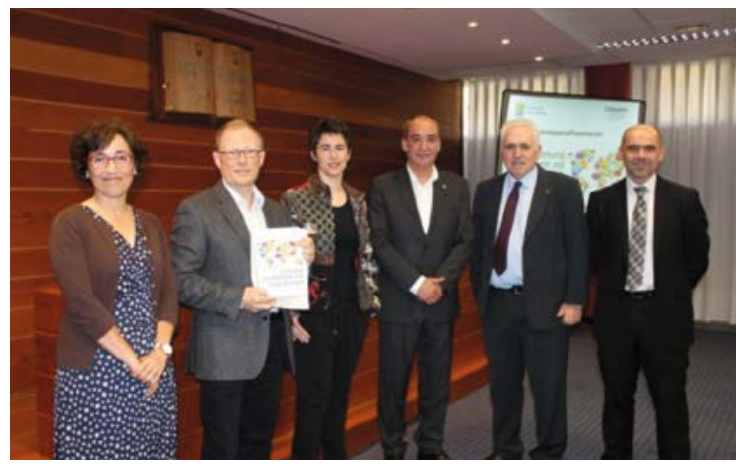
trascendido, en parte, a la comunidad académica a través de publicaciones realizadas por los investigadores (véase Anexo 1).

No obstante, los intentos para llegar a policy makers que no participaron en el proceso han sido escasos y poco focalizados. Se hizo el esfuerzo de hacer una publicación divulgativa ${ }^{2}$ que recogiera las reflexiones de todos los actores que han participado en el proceso, así como una guía para la plataforma RRI-Tools ${ }^{3}$, que busca recoger prácticas de investigación e innovación responsables (RRI, por sus siglas en inglés) en Europa.

Por todo ello se considera que el presente documento, planteado como guía para policy makers que se enfrentan al reto de innovar en las políticas integrando en las mismas a actores del territorio, es un documento relevante que cubre un vacío. El objetivo en este sentido es compartir el conocimiento desarrollado de una manera que pueda ser útil en el día a día de los policy makers tanto en el ámbito local (ayuntamientos), territorial (diputación) y Gobierno Vasco (comunidad autónoma). Para lograr este objetivo, se ha utilizado material que se ha ido sistematizando a lo largo de estos años y que permite compartir no sólo el modelo teórico construido, sino las vivencias de los participantes en el proceso.

\footnotetext{
${ }^{2}$ Gipuzkoako Foru Aldundia (2015). Gipuzkoa Sarean. Bidean. Gipuzkoako Foru Aldundia: Donostia - San Sebastián.

${ }^{3}$ Building Bridges: A four step framework to improve territorial governance.
} 


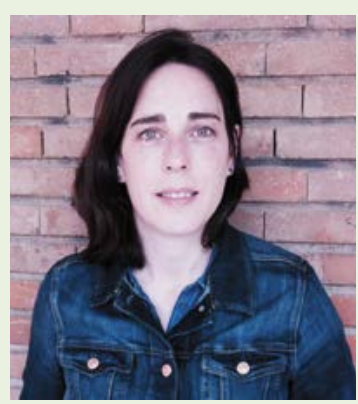

«Todo el proceso de Gipuzkoa Sarean está documentado. Según Guba y Lincoln, una forma de que aquellos que no participaron en un proceso aprendan del mismo es proveerles de una vivencia indirecta, es decir, compartir no sólo lo que ocurrió, sino también las razones y motivaciones que había detrás».

Ainhoa Arrona, Orkestra, miembro del equipo de investigación de Gipuzkoa Sarean entre 2009 y 2015.

\section{Guía para la innovación en políticas de desarrollo territorial}

Tal y como se adelantaba en la introducción, el proceso de Gipuzkoa Sarean se puede sintetizar en un modelo de trabajo que ha sido fruto de la co-generación, es decir, de la combinación de distintas formas de conocimiento (académico, práctico) y también de la reflexión y la acción. Las fases del modelo se muestran en la Figura 1.

\section{FIGURA 1. Fases del proceso de innovación en las políticas}

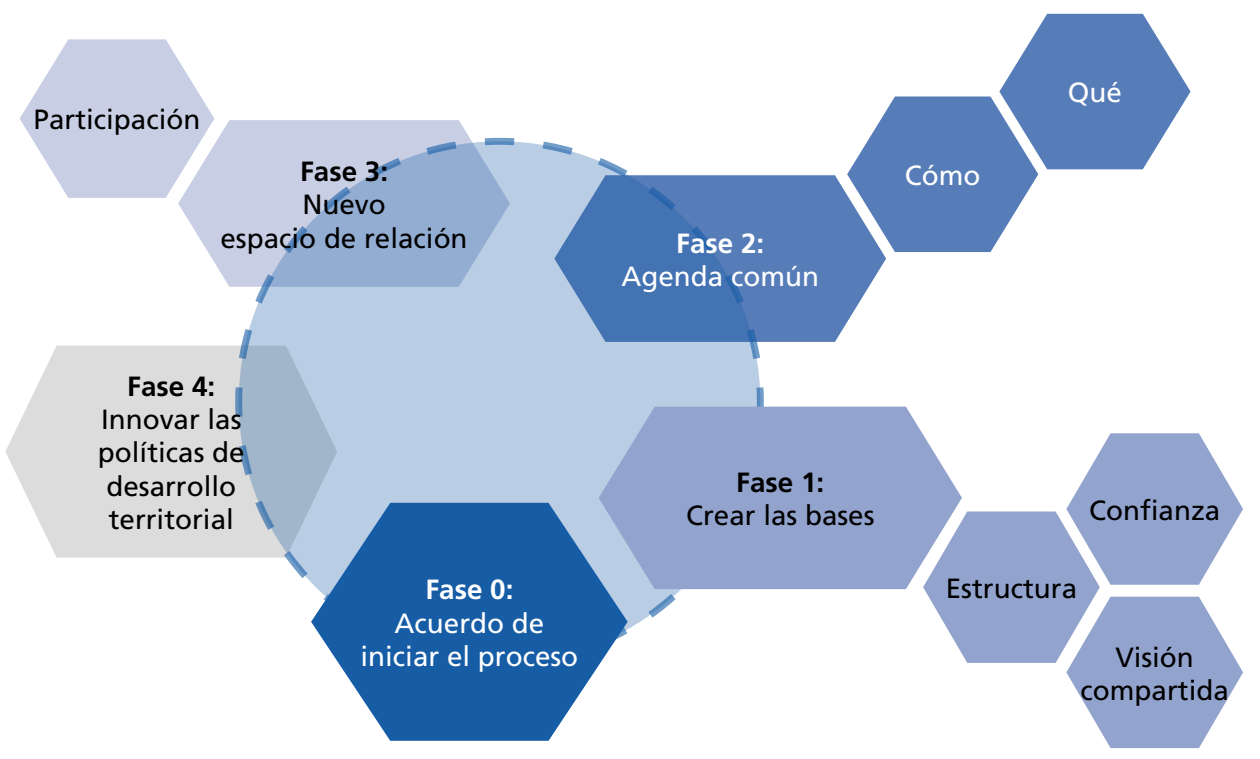




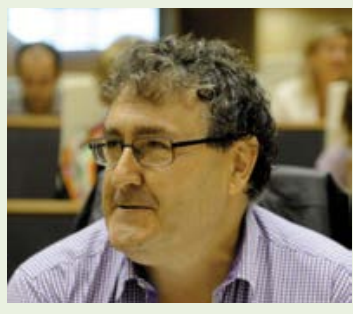

"Los investigadores han llevado a cabo un proceso de resistencia suave pero continuo. El proceso es cíclico, reflexión-política, reflexión-política, pero es un círculo que no es estático, avanza como una rueda».

Juan Karlos Alduntzin, jefe de gabinete de la Diputación Foral de Gipuzkoa entre 2013 y 2015.

La Figura 1 recoge las fases necesarias para desarrollar un proceso de innovación en políticas. No obstante, conviene destacar que no han de entenderse como fases lineales, sino como un proceso cíclico en el que las fases posteriores alimentan a las anteriores y en el que las mismas a veces se solapan. En las siguientes secciones se describe cada una de estas fases.

\section{FASE 0: Acuerdo de iniciar el proceso: el reto de encontrar un modelo para la colaboración entre policy makers e investigadores}

Lo importante en esta fase es negociar y acordar entre policy makers e investigadores cuál es el problema que se quiere resolver (qué) y cuál es el proceso mediante el cual se va a resolver (cómo).

Para negociar el cómo, es importante que los investigadores planteen un marco analítico del proceso. La Figura 2 (Karlsen y Larrea, 2014) presenta el marco que representa este proceso en la Investigación Acción para el Desarrollo Territorial.

El primer elemento sobre el que debe tomarse conciencia de forma conjunta entre policy makers e investigadores es que el proceso se va a desarrollar en un contexto de complejidad territorial. Ello implica que habrá diversidad de actores con interpretaciones potencialmente distintas de cuáles son los principales problemas a resolver por las políticas y las potenciales soluciones a los mismos. Al no haber en el territorio un actor con una posición jerárquica que permita imponer las soluciones (esto no significa que haya actores con más poder que otros), las soluciones no podrán desarrollarse a través del ordeno y mando. Es esto último lo que hace que necesariamente deban buscarse soluciones dialogadas.

El modelo plantea también la importancia de que al inicio del proceso, tanto policy makers como investigadores, definan el ágora como espacio de encuentro entre ellos. En este espacio la política se encuentra con la academia y los planteamientos de unos y otros se van haciendo más robustos al someterse al filtro tanto de las políticas a través de la reflexión académica, como del conocimiento académico al someterse a la necesidad de aportar valor a las políticas. 


\section{FIGURA 2. Creación de conocimiento colectivo en la acción}
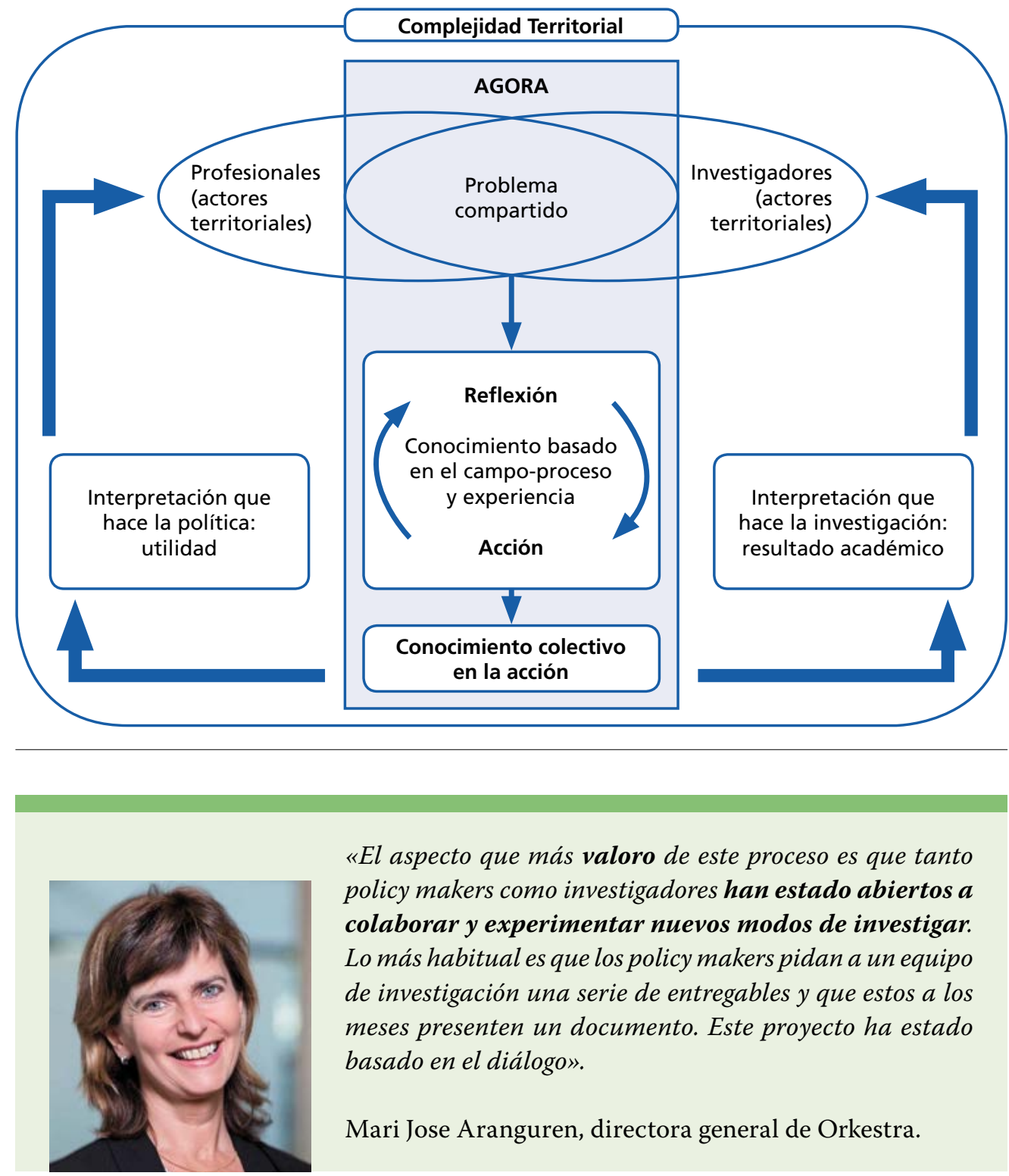

«El aspecto que más valoro de este proceso es que tanto policy makers como investigadores han estado abiertos a colaborar y experimentar nuevos modos de investigar. Lo más habitual es que los policy makers pidan a un equipo de investigación una serie de entregables y que estos a los meses presenten un documento. Este proyecto ha estado basado en el diálogo».

Mari Jose Aranguren, directora general de Orkestra. 
Como ya señalamos, en esta fase inicial además de consensuar el modelo sobre el proceso, es importante consensuar el problema a resolver. Ello aparece en el modelo como problema compartido, que debe entenderse como resultado de un proceso de negociación en el que policy makers e investigadores pueden tener intereses distintos. Es necesario entender que no se trata exclusivamente de un proceso de investigación orientado a generar nuevo conocimiento, ni de un proceso de construcción de políticas orientado a resolver un problema. Es ambas cosas a la vez, y este equilibrio debe acordarse desde el principio.

Una vez definido el problema compartido, lo que el modelo plantea es iniciar procesos de reflexión-acción, en que los policy makers ponen encima de la mesa los problemas que tienen en su proceso y los investigadores contribuyen con marcos y conceptos a mirar el problema desde ángulos que permitan ir construyendo soluciones. Las decisiones sobre las políticas son siempre responsabilidad de los policy makers y en la medida en que estos pasan a la acción a través de pequeñas decisiones, el proceso avanza. De esta manera se van abordando redefiniciones del problema anterior o si este se hubiera resuelto, se definen problemas nuevos.

Mantener estos procesos en el tiempo da como resultado la generación de conocimiento colectivo en la acción. El conocimiento colectivo en la acción es una capacidad, un patrón aprendido de acción colectiva, según el cual los actores de un ágora modifican sistemáticamente sus acciones a lo largo del tiempo, a través de un proceso de aprendizaje. Este tipo de conocimiento es una capacidad que solo puede desarrollarse mediante el proceso de aprendizaje que tiene lugar cuando un grupo de actores del territorio se reúne regularmente y cuando sus miembros interactúan entre ellos. Esto se persigue mediante el diálogo. Mediante el diálogo tienen lugar conexiones entre conceptos teóricos, discursos y situaciones reales que crean la base para la acción y para cambiar una situación determinada en un territorio. En estos espacios de diálogo, los conceptos teóricos se someten a discusión y se convierten en acciones concretas. El diálogo es un método para provocar cambios en el lenguaje, en la conducta y en estructuras organizativas e institucionales.

Lo que no se puede trasladar con el modelo anterior es el nivel de compromiso que el mismo requiere. La mejor manera de ilustrarlo es a través de las palabras de los policy makers participantes. 


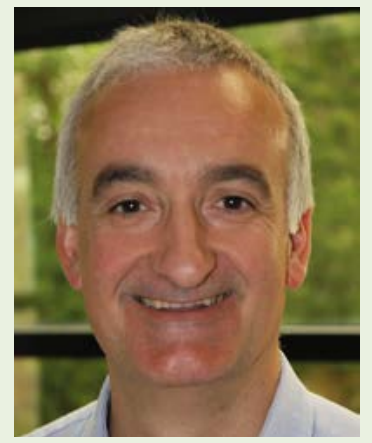

«Soy consciente de que los procesos compartidos entre policy makers e investigadores pueden fallar. Creo que para preservar este tipo de procesos y obtener los resultados esperados son necesarias cuatro condiciones. La primera, una garantía política en el sentido de que se respetará el resultado del proceso. La segunda, la participación de aquellos que pueden decidir y aquellos que tienen el conocimiento. Esto está relacionado con el poder, ya que aquellos que pueden decidir ceden parte de su poder a favor de un proceso determinado. La tercera es que los participantes, ya sean policy makers o investigadores, tiene que dejar de lado ambiciones personales para priorizar los objetivos del proyecto. Finalmente, la cuarta condición es que tiene que existir una admiración personal entre policy makers e investigadores: algo que trasciende las buenas maneras».

Xabier Barandiaran, responsable de Gabinete de la Diputación Foral de Gipuzkoa y director de Gipuzkoa Sarean entre 2009 y 2011.

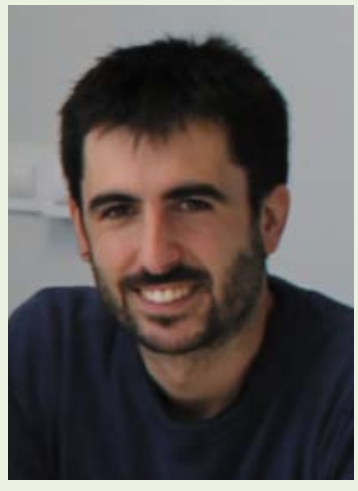

«La participación de los investigadores ha sido totalmente positiva, pero ha requerido un constante compromiso. La investigación requiere reflexión y para la reflexión hace falta tiempo. La metodología empleada exigía una reunión semanal, y esto supone un esfuerzo. Hace falta esfuerzo y compromiso respecto a la metodología y fidelidad al proceso. Mantener dicho compromiso no es fácil».

Ugaitz Iturbe, miembro de la Dirección para el Desarrollo Territorial de la Diputación Foral de Gipuzkoa entre 2013 y 2015.

La manera en la que se aborda la definición del problema es crítica para la creación de conocimiento colectivo en la acción. Es el primer consenso que por frágil que pueda parecer es indispensable para abordar las siguientes etapas. Los consensos dan credibilidad al proceso porque son fruto de decisiones y acciones. La aproximación de los actores al problema determina la implicación y, con ello, la posibilidad de contribuir a la solución del problema. 


\section{FASE 1: Creación de las condiciones básicas para el proceso de innovación en políticas}

¿Cómo podemos crear las bases necesarias que garanticen la consistencia del proceso? ¿En qué aspectos tienen que incidir los policy makers y los investigadores para asentar estas bases? El principal argumento es que el conocimiento colectivo en la acción es el resultado de construir capital social en sus diferentes dimensiones. Este conocimiento no es el resultado de una transferencia lineal de aquellos que conocen la respuesta a un problema a aquellos que tienen el problema. Es decir, no estamos hablando de un modelo en el que el investigador encuentra primero las soluciones a los problemas del policy maker y después, plantea sus recomendaciones, sino que son procesos en que juntos cogeneran dichas soluciones. Para poder abordar un proceso de este tipo hay tres condiciones básicas. Intentar avanzar en el proceso sin haber generado estas condiciones es uno de los factores que hace que los procesos se estanquen. Las tres condiciones son: crear los espacios de diálogo, construir las relaciones de confianza y construir la visión compartida sobre el proyecto.

\section{a. Crear los espacios de diálogo}

Mediante el diálogo tienen lugar conexiones entre conceptos teóricos, discursos y situaciones reales que crean la base para la acción y para cambiar una situación determinada en un territorio. En estos espacios de diálogo, los conceptos teóricos se someten a discusión y se convierten en acciones concretas. El diálogo es un método para provocar cambios en el lenguaje, en la conducta y en estructuras organizativas e institucionales. Las siguientes preguntas pueden ayudar a crear espacios de diálogo:

- ¿Cuál es el estatus de los participantes (el rol que juegan) y en consecuencia, su capacidad de decisión?

- ¿Todos los participantes han interiorizado su estatus?

- ¿El estatus de cada uno está reconocido por todos?

\section{b. Construir las relaciones de confianza}

El mejor catalizador del diálogo es la confianza. Un diálogo se asienta en la confianza que los que dialogan tienen entre sí, porque entienden que tienen algo valioso que aportar el uno al otro. La confianza se crea cuando hay coherencia entre el discurso y la práctica, cuando se percibe una escucha sincera, más allá de estar de acuerdo, o no, con lo que se dice. El diálogo se basa en la interdependencia y reconocimiento de la otra parte. 


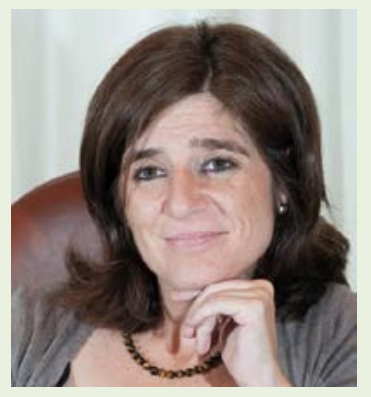

«La participación y la confianza son aspectos básicos. Entre los actores del territorio, especialmente entre actores políticos, hay una gran falta de confianza, y además el sistema electoral que tenemos insiste en destacar las diferencias. Deberíamos trabajar modelos de participación y compartir proyectos. Trabajar este ámbito es necesario para poder abordar cualquier otro proyecto».

Helena Franco, diputada de Hacienda de la Diputación Foral de Gipuzkoa entre 2011 y 2015.

La confianza no es automática, es necesario trabajarla. No puede pedirse ni imponerse. Por eso, los espacios de innovación en políticas requieren un trabajo previo, un trabajo de cocina a fuego lento, donde ir generando la confianza.

Muchas veces la estrategia para construirla es pasar rápido del discurso a pequeñas acciones en el territorio, que sirven mucho más que el discurso para generar confianza. Una pequeña acción llevada a cabo con éxito genera confianza, porque permite en la siguiente ronda, abordar una acción un poco más ambiciosa. Otras veces es necesario generar espacios transparentes donde la información fluya, otras trabajar para que se cumplan acuerdos.

Las siguientes preguntas pueden ayudar a reflexionar sobre si se ha creado un espacio de confianza:

- ¿Confían los participantes en que todos tienen buenas intenciones?

- ¿Confían los participantes en las capacidades del resto?

- ¿El proceso es transparente?

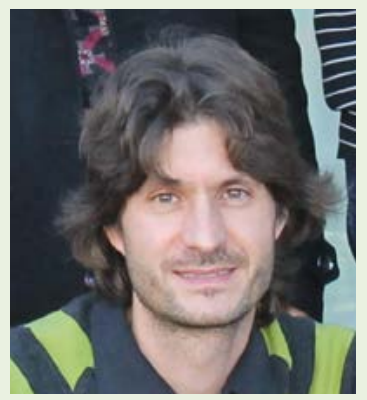

«En Gipuzkoa Sarean hemos podido ver la importancia de las relaciones en los proyectos e iniciativas, y cómo el grado de confianza influye en los resultados y en la forma de encaminar los procesos. Gracias a la flexibilidad del proceso, hemos sido capaces de corregir y mejorar en el transcurso del mismo».

Xabier Sanchez, Agencia de Desarrollo Comarcal Oarsoaldea. 


\section{c. Construir la visión compartida}

Los espacios de diálogo responden a una necesidad cuya complejidad requiere de una respuesta trabajada de forma colectiva. La complejidad de los problemas que se abordan a nivel territorial, no se resuelve desde la mentalidad de proyecto a corto plazo, es necesaria una perspectiva de proceso a largo, de generación de un cambio evolutivo. Cuando nos referimos a procesos de innovación en políticas es necesario, por lo tanto, gestionar las expectativas en el corto plazo. Esto requiere una lectura crítica de las dinámicas que han llevado a la situación presente. El objetivo del diálogo en este contexto es generar decisiones a corto que estén alineadas con la construcción a largo plazo.

En la búsqueda de este equilibrio entre el largo y corto plazo, en Gipuzkoa Sarean se interpretó que la visión compartida no consistía en que todos llegaran a pensar lo mismo. La visión compartida se definió como la interpretación compartida de las diferencias. Es decir, entre todos se intentó construir un relato sobre las distintas interpretaciones existentes entre los participantes en relación con los problemas existentes y sus posibles soluciones. Esta interpretación compartida de las diferencias generaba mejores condiciones para buscar los espacios (a veces nichos) de acuerdo para la acción.

Las siguientes preguntas pueden ayudar a reflexionar sobre si se ha generado una visión compartida:

- ¿Pueden los participantes explicar en qué consiste el proceso?

- ¿Qué diferencias existen en el modo en el que los participantes interpretan el objetivo general del proceso y la filosofía en la que descansa su desarrollo?

- ¿Existen elementos mínimos compartidos por todos?

\section{FASE 2: Definición de una agenda compartida}

Una vez generadas las condiciones básicas anteriores, se puede proceder a definir una agenda compartida. Esta agenda debe abordar con claridad qué se quiere conseguir con el proceso de co-generación, pero debe especificar también cómo se quiere conseguir.

El cómo es uno de los grandes olvidados en los procesos de innovación en políticas. 


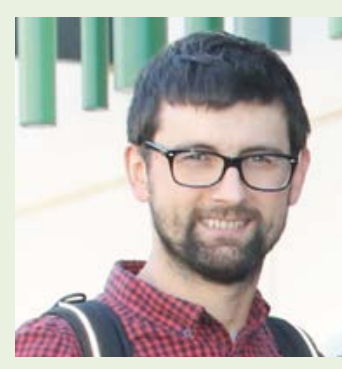

«La aportación más novedosa de Gipuzkoa Sarean es la reflexión en torno al proceso. En un proceso siempre existe un 'qué' y un 'cómo', y a mi parecer, dar importancia al 'como' es indispensable. Esto es lo que nos enseña Gipuzkoa Sarean».

Borja Urretabizkaia, facilitador de la Agencia de Desarrollo Comarcal Goierri.

La colaboración, que frecuentemente se plantea como mecanismo para la innovación en las políticas, no es un proceso fácil en el que todos colaboran porque tienen buenas intenciones. La colaboración es en realidad un proceso de gestión de los conflictos que emergen porque los actores participantes tienen interpretaciones distintas de cuáles son los problemas del territorio y sus posibles soluciones. Es por eso que en este apartado se dedica un espacio significativo al conflicto.

\section{El conflicto es necesario para} generar nuevos contextos que permitan nuevas interacciones a partir de consensos

Superar el conflicto no significa unificar perspectivas, sino aprender a integrar las diferencias en la acción. Es por eso que el proceso no es lineal. Es difícil a priori determinar el resultado de un proceso de construcción en red, cuando el proceso depende y requiere de las aportaciones de los participantes.

Uno de los puntos críticos en el impulso al desarrollo territorial es entender que las etapas de conflicto son algo natural, y no algo que ha de evitarse. Se trata de relativizar y normalizar el conflicto como algo inherente a la complejidad de las relaciones humanas. Es necesario aceptar esta realidad para poder construir nuevas perspectivas, conocimiento e innovaciones. El conflicto representa crisis y oportunidad.

Conviene tener en cuenta que para gestionar un conflicto es necesario explicitarlo y que ello tiene un coste, pues genera tensiones y dificulta las relaciones. En general, para que un actor del territorio esté dispuesto a hacer explícito un conflicto tiene que tener una expectativa de que existen posibilidades para resolverlo y si esto se consigue, los beneficios serán superiores al coste. La Figura 3 (Karlsen y Larrea, 2014) presenta un marco para la reflexión que puede resultar útil como una herramienta de análisis para entender las situaciones y tratar de resolverlas. 


\section{FIGURA 3. Tipos de complejidad territorial}

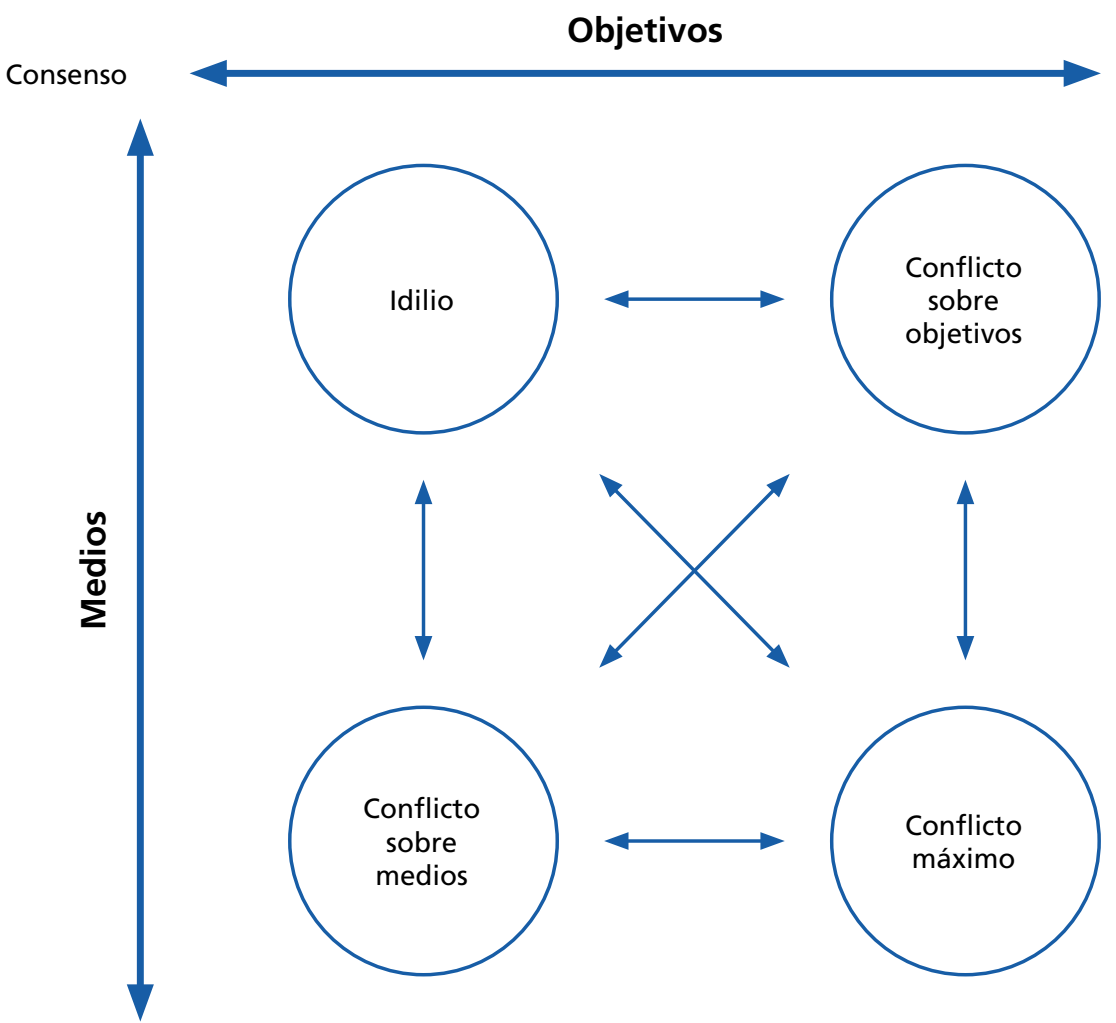

Conflicto

En la mayoría de procesos de innovación en las políticas que no avanzan según las expectativas, existe algún tipo de conflicto, bien sobre los objetivos del proceso o bien sobre los medios (recursos económicos, roles, etc.). La explicitación de dichos conflictos y su gestión debe llevar el proceso a un punto de consenso.

Cuando un proceso alcanza un punto de consenso, por frágil que sea, se abre una ventana de oportunidad para actuar, lo que podría cambiar el equilibrio nuevamente. Con un nuevo equilibrio pueden llegar nuevos conflictos, lo que genera la necesidad de continuar con el diálogo. Por eso debe mantenerse la perspectiva de proceso cíclico en el que de vez en cuando se generan las condiciones para actuar.

A continuación se describen cinco etapas que ilustran el desarrollo del conflicto en un proceso de innovación en políticas de desarrollo territorial. 


\section{a. Generar conciencia del conflicto}

Aquellas situaciones en las que existe un consenso aparente o un consenso en los grandes objetivos, pero en las que no sucede nada concreto, normalmente tienen un conflicto tácito. Una de las funciones del investigador es ayudar a los actores a detectarlo.

En un proceso cogenerativo el policy maker puede, por lo tanto, recurrir a los investigadores para reflexionar sobre cuáles son los principales conflictos tácitos existentes en el proceso.

\section{b. Explicitar el conflicto}

Otra de las funciones del investigador es ayudar a los actores a explicitar el conflicto de una manera constructiva. No hay recetas que puedan garantizar soluciones al abordar el conflicto. Sin embargo es importante diseñar unas reglas anticipando que los diferentes actores tendrán perspectivas e intereses distintos y generando los mecanismos a través de los cuáles podrán explicitarse estas diferencias.

Los marcos y conceptos que el investigador aporta para crear el lenguaje compartido pueden ayudar a hacer explícito el conflicto ya que los participantes pueden empezar a hablar del problema a un nivel más teórico para posteriormente ir compartiendo la concreción de los mismos.

\section{c. Afrontar el conflicto entre red y jerarquía}

La metodología planteada está orientada al desarrollo de políticas participativas e integradoras de los actores territoriales, que normalmente apuestan por el desarrollo de redes de colaboración. Es importante no olvidar que, aunque las redes planteen estructuras planas en que se superan los modelos jerárquicos, siempre habrá una incidencia de las jerarquías que influyan en el proceso, tanto porque las jerarquías dentro de las organizaciones influyen en cómo los representantes de dichas organizaciones colaboran, como porque no todos los actores participantes tienen el mismo estatus en el proceso. 
Los investigadores pueden jugar en el proceso un papel de facilitadores que ayuda a que las relaciones en red puedan ir superando las diferentes culturas de la colaboración y las diferencias en estatus de los actores participantes.

\section{d. Mantener vivo el diálogo}

En todo proceso hay momentos de crisis en que no es fácil mantener el diálogo. La aportación del investigador en estos contextos es crear nuevas oportunidades para el diálogo presentando conceptos y marcos conceptuales que puedan ayudar a evolucionar en direcciones nuevas evitando el estancamiento.

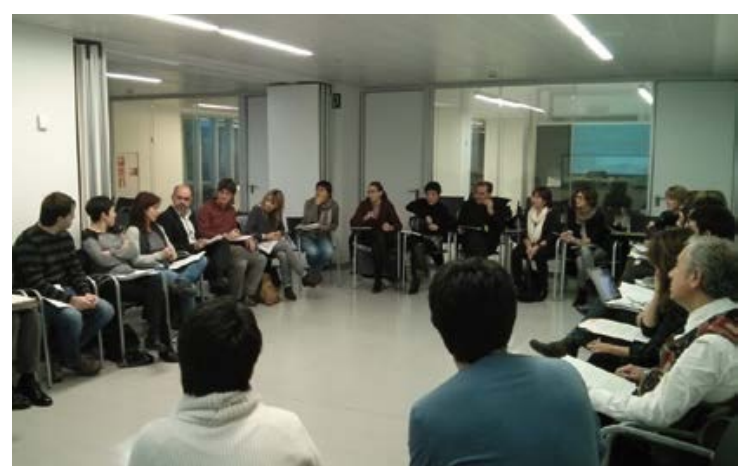
Los espacios formales son importantes y es decisivo diseñarlos adecuadamente para permitir el diálogo. Pero en un proceso de largo plazo habrá momentos en que el diálogo tenga lugar también en espacios informales.

Para poder mantener el diálogo, es necesaria la participación del investigador no sólo en los espacios formales, sino también en los informales. La confianza del policy maker en el investigador es lo que permite que éste tenga acceso a estos últimos.

e. Consolidar una estrategia de investigación acción para la innovación en políticas

El papel del investigador no es proponer una solución teórica final que supere el conflicto, es dotar a los participantes de conceptos y marcos conceptuales que sean útiles para hacerlo en la práctica. Aunque la solución cogenerada en la práctica pueda parecer imperfecta desde el punto de vista teórico, el resultado tiene una mayor posibilidad de implementación real. 


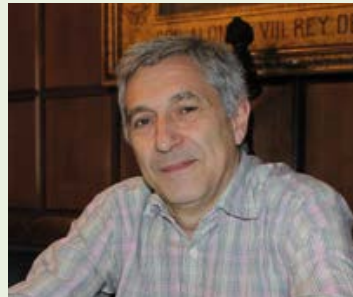

«Los investigadores no nos dicen qué deberíamos hacer, nos ayudan a reflexionar y somos nosotros quienes construimos nuestras propias soluciones».

Sebastián Zurutuza, director de Estrategia de Gestión de la Diputación Foral de Gipuzkoa y director de Gipuzkoa Sarean desde 2015.

La investigación acción para el desarrollo de políticas requiere del investigador un papel al que no siempre está acostumbrado, pues no se le solicitan recomendaciones para las políticas, sino acompañar a los policy makers en la búsqueda de sus propias soluciones. Este cambio no depende sólo de los investigadores, sino que depende en gran medida del desarrollo de una demanda en este sentido por parte de los policy makers.

\section{FASE 3: Construcción de un nuevo modelo de relación}

Una vez establecida la agenda compartida sobre qué se quiere hacer y cómo, llega el momento de pasar a la acción. Este es el punto en que muchos de los procesos de innovación en las políticas se estancan. Es difícil pasar del papel a las acciones.

Tener una agenda compartida no significa que ya se cuente con un nuevo modelo de gobernanza que permita innovar en las políticas. Es en el desarrollo práctico de la agenda cuando se construye de verdad la nueva gobernanza. En este punto el modelo sigue planteando el diálogo como herramienta de trabajo. Pero el diálogo es mucho más que conversación, tiene que tener una relación con la práctica para plasmar lo hablado en un contexto real. El cambio es fruto de procesos de diálogo porque mediante el lenguaje se construyen nuevas interpretaciones de la realidad que llevan a nuevas conductas y redundan finalmente en nuevas prácticas. Los cambios en los patrones de comunicación son la punta de lanza en el proceso de cambio.

Los cambios no ocurren de forma espontánea. Siempre que analizamos los procesos de cambio encontramos personas detrás que los hicieron posible, personas que empujaron el proceso. Se trata de los facilitadores. La facilitación es una forma de trabajar, y se expresa en las acciones que favorecen contextos para la interacción, para el diálogo. Este rol no recae directamente sobre una persona concreta. Las 
acciones que promueven la facilitación pueden estar repartidas en distintos momentos en personas diferentes.

La facilitación en el desarrollo territorial es importante porque el desarrollo territorial se da en contextos de conflicto. La labor

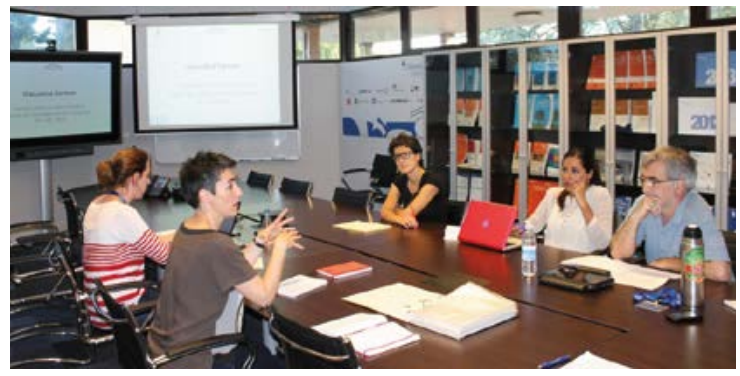
del facilitador es crear las condiciones para que los actores, mediante el diálogo, puedan llegar al consenso y pasen a la acción. Hay momentos en los que la facilitación requiere un liderazgo muy sólido. Sin embargo, el liderazgo del facilitador no es un liderazgo jerárquico, sino relacional, construido a base del reconocimiento de los actores, construido desde la práctica y en el día a día.

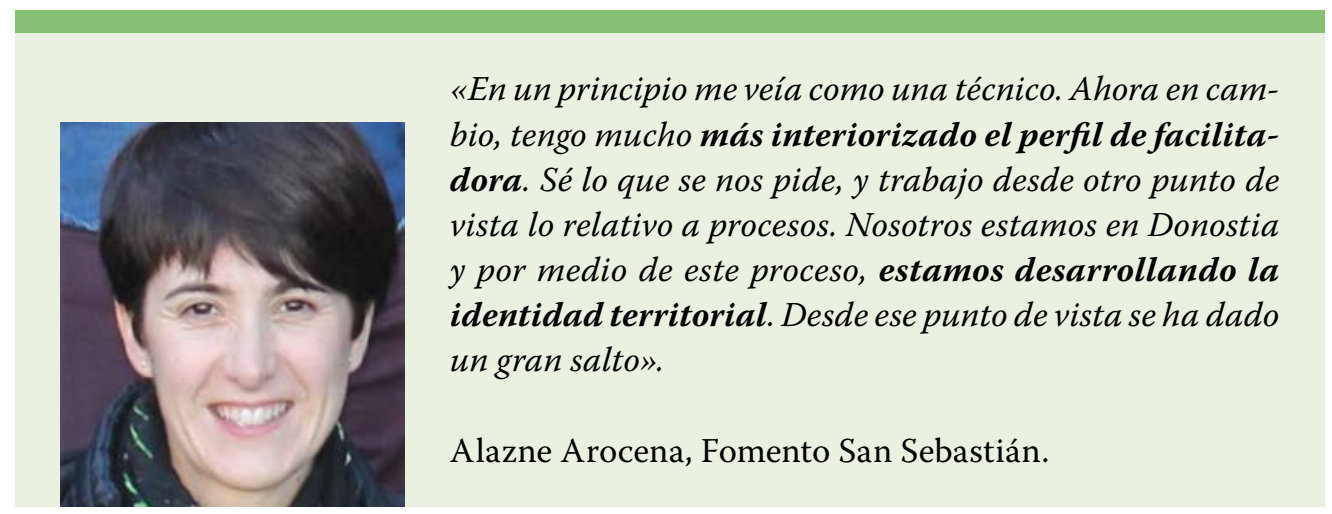

FASE 4: La innovación en las políticas como resultado del proceso

La innovación en las políticas, emerge de los anteriores procesos de diálogo, en los que la mutua influencia hace que vayan cambiando las actitudes y los comportamientos y se vayan alineando las actuaciones.

El resultado de la co-generación depende del acuerdo inicial entre policy makers e investigadores y del desarrollo del proceso. Como consecuencia, los resultados serán distintos en cada caso. Sin embargo, un elemento común es que dicho resultado final difícilmente puede ser anticipado en su concreción, pues se construye de manera emergente. Es decir, todo el proceso está orientado a un resultado que, al construirse de forma participativa, no puede ser descrito a priori. Otro elemento común es que normalmente esta construcción conjunta requiere cambios en los modelos de relación, en la gobernanza de las políticas. 
En el caso de Gipuzkoa Sarean la innovación en políticas se planteaba en términos de construcción de políticas de forma participativa entre la Diputación Foral de Gipuzkoa y las agencias. Los resultados concretos fueron los decretos que ya se mencionaron al inicio de este documento. Sin embargo, la nueva gobernanza construida sigue siendo un caldo de cultivo para nuevas iniciativas que permitan seguir innovando.

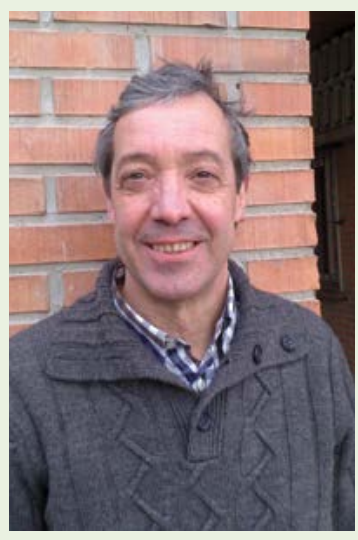

«La mesa comarcal es un ejemplo claro del nuevo modelo de gobernanza, diría yo. Se quiere construir una nueva Gipuzkoa integrando la realidad y los retos de los actores comarcales, con una visión territorial.

Cuando se puso en marcha, se hacía difícil pronosticar el resultado, porque siendo un proceso abierto, no habia ningún camino establecido, y por supuesto, menos el resultado, solo la dirección. Más que el resultado, diría que importa el proceso, los aprendizajes que hemos adquirido en el proceso, las relaciones, las diferentes realidades y prioridades comarcales, la perspectiva territorial».

Jesus Agirre, director de la Agencia Comarcal Iraurgi Berritzen.

\section{Conclusiones}

La sociedad vive un momento de cambios que obliga tanto a los distintos niveles de gobierno como a la universidad a adaptarse e integrar modos de trabajo más participativos que los acerquen a la sociedad. Este briefing presenta una forma de trabajo cogenerativo que permite cumplir con este objetivo. Por una parte, permite a los distintos niveles de gobierno y, por lo tanto, a los policy makers, construir espacios de diálogo con otros actores del territorio para desarrollar espacios de deliberación para las políticas. Por otra, al integrar a los investigadores en dichos procesos como facilitadores, permite multiplicar el impacto social de la investigación realizada.

Establecer condiciones para la co-generación no es una tarea simple. Requiere procesos de construcción de confianza y visión compartida, y para ello, hay que crear primero los espacios de diálogo adecuados. Todo ello conlleva invertir en una serie de intangibles cuyos resultados sólo se visibilizan en el largo plazo. Es 
decir, estas aproximaciones requieren una apuesta, no sólo por parte de los policy makers, sino también de los investigadores.

No era objetivo de este briefing evaluar el proyecto de investigación que ha dado lugar a los conocimientos compartidos en el mismo. Sin embargo, consideramos que las valoraciones que del proceso se han recogido de la mano tanto de policy makers como de investigadores, animan a considerar que este es un camino costoso pero interesante para aquellos que buscan reformular las políticas a través de la investigación y la investigación a través de las políticas.

\section{Referencias}

Karlsen J. y Miren Larrea. Territorial Development and Action Research: Innovation Through Dialogue. Gower: Surrey, 2014. 


\section{Notas biográficas}

\section{Patricia Canto}

Trabaja desde hace una década en el área de comunicación de Orkestra-Instituto Vasco de Competitividad. Entre sus responsabilidades destacan la elaboración de la memoria de actividades que se distribuye todos los años a los principales stakeholders del Instituto, la coordinación editorial del Informe de Competitividad del País Vasco, publicación que Orkestra presenta a la sociedad cada dos años. Actualmente está realizando su tesis doctoral, en la que explora el vínculo entre comunicación e impacto social de la investigación. Antes de trabajar en Orkestra trabajó en el Ministerio de Economía de México. Ha sido docente en la Universidad de Deusto y en el Instituto Tecnológico de Estudios Superiores de Monterrey (México).

\section{Ion Irurzun}

Se incorporó recientemente como facilitador de investigación en Orkestra-Instituto Vasco de Competitividad con el objeto de poner en valor el papel del facilitador y el valor añadido que este incorpora en los procesos de investigación. Su trayectoria profesional tiene una marcada orientación a la facilitación en procesos de cooperación y es en este ámbito donde se ha focalizado los últimos años dentro del proyecto Gipuzkoa Berritzen, cuyo propósito era la promoción de la innovación en cooperación. Su experiencia es esencialmente práctica en la formación y desarrollo de la red, como procesos de cooperación, poniendo el foco en el conflicto como condicionante de la evolución del proceso.

\section{Miren Larrea}

Es investigadora senior en Orkestra-Instituto Vasco de Competitividad, docente en la Universidad de Deusto y colaboradora regular en la Universidad Tecnológica Nacional, Facultad Regional Rafaela (Argentina) y la Universidad de Agder (Noruega). Sus áreas de especialización en la investigación son los procesos de aprendizaje en las políticas, el desarrollo local, la gestión de redes y los modos de gobernanza que facilitan la colaboración. Cuenta con experiencia práctica en el desarrollo de redes locales para el aprendizaje en cooperación y con publicaciones tanto a nivel local como internacional en los ámbitos señalados. Coordina diversos proyectos con gobiernos municipales y regionales del País Vasco que le han permitido construir la aproximación a la investigación acción para el desarrollo territorial, uno de los ejes de la estrategia de Orkestra para la investigación transformadora. 


\section{Anexo 1. Publicaciones académicas derivadas del proyecto Gipuzkoa Sarean}

Barandiaran, Xabier y Kepa Korta. Capital social y valores en Gipuzkoa. Diputación Foral de Gipuzkoa. Donostia - San Sebastián: Diputación Foral de Gipuzkoa, 2011.

Costamagna, Pablo. Política y formación en el desarrollo territorial. Bilbao: Publicaciones Deusto, 2015.

Karlsen, James y Miren Larrea. «Moving context from the background to the forefront of policy learning: Reflections on a case in Gipuzkoa, Basque Country» Environment and Planning C: Government and Policy (2016): 1-16. doi: 10.1177/0263774X16642442.

Karlsen J., y Miren Larrea. «Interaction between Research and Practice: Editorial». International Journal of Action Research, 10, $\mathrm{n}^{\circ} 2$ (2014): 125-128.

Karlsen J. y Miren Larrea. Territorial Development and Action Research: Innovation Through Dialogue. Gower: Surrey, 2014.

Karlsen J. y Miren Larrea. «The contribution of Action Research to Policy Learning: The Case of Gipuzkoa Sarean». International Journal of Action Research, 10, n 2 (2014): 129-155.

Larrea, Miren. «¿Hacia dónde evoluciona la participación para el desarrollo local? Reflexiones desde Gipuzkoa». En Ciudadanía Empoderada: cultura y participación para el desarrollo loca, editado por Ricardo Calvo, Juan A. Rodríguez, A., Jaume Portet; Mónica Bou y José M. Romera. Valencia: Germania, s.I., 2013.

Larrea, M., María José Aranguren, y James Karlsen. «The Policy Process in Regional Innovation Systems: Targeting Behavioural Value Added of Policy in Gipuzkoa, Basque Country". En Perspectives on Global and Territorial Change, editado por Philip Cooke y Mario. D. Parrilli. Londres: Palgrave, 2012. 



\title{
Guidelines for innovation in public-sector policies: maximising research potential
}

\author{
Patricia Canto, Ion Irurzun, Miren Larrea
}

1. Introduction: why produce guidelines for innovation in public-sector policies through research? ................... 36

2. Rationale and Context.......................... 39

3. Guidelines for innovation in territorial development policies ...... 40 STAGE 0: Beginning the process: the challenge of finding a model for co-operation between policy makers and researchers . . . . . 41 STAGE 1: Creating the basic conditions for the process of innova-

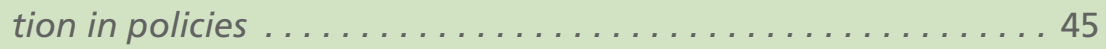

STAGE 2: Defining a shared agenda ................. 47

STAGE 3: Constructing a new space for relations ............ 52 STAGE 4: Innovation in policies as an outcome of the process. . . . . 53

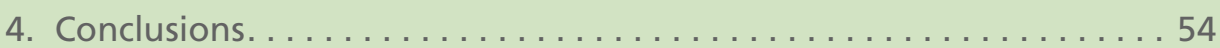

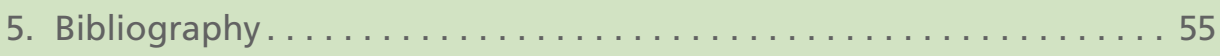

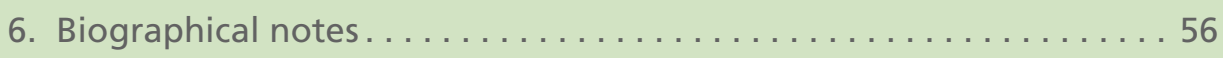

Annex 1. Academic publications arising from the Gipuzkoa Sarean project .................................. 57 



\section{Guidelines for innovation in public-sector policies: maximising research potential}

Patricia Canto, Ion Irurzun, Miren Larrea

doi: http://dx.doi.org/10.18543/dsib-1(2016)-pp173-195.pdf

\section{Abstract}

This document sets out guidelines for innovation in public-sector policies through action research processes. It seeks to convey to policy makers the idea that by implementing this working method with research teams they can generate spaces for understanding the problems that exist in policy development processes. It can also serve to generate new frameworks and concepts for thinking about those problems differently, and may thus facilitate decision-making processes that can be implemented incrementally as learning progresses.

\section{Keywords:}

Innovation, policies, action research, dialogue, trust

\section{Resumen}

El documento ofrece una guía para la innovación en las políticas públicas a través de procesos de investigación acción. Plantea a los policy makers que estableciendo este método de trabajo con un equipo de investigación pueden generar espacios en que se tome conciencia de los problemas existentes en los procesos de desarrollo de políticas, se generen nuevos marcos y conceptos para pensar dichos problemas de maneras distintas y se faciliten procesos de decisión que se van implementando de forma incremental, en la medida en que se va aprendiendo.

\section{Palabras clave:}

innovación, políticas, investigación acción, diálogo, confianza 


\section{Introduction: why produce guidelines for innovation in public-sector policies through research?}

In the field of regional policies there is increasing recognition of the importance of incorporating new actors into forums for discussion and into policy decision-making. However, the processes implemented have proved so difficult that they often stagnate. Here we seek to determine what those difficulties are and how they can be overcome.

These guidelines are based on the learning outcomes of an action research project called "Gipuzkoa Sarean», carried out between 2009 and 2016. The aim is to help overcome that stagnation and put into practice conceptual frameworks that are widely recognised at a theoretical level but have proved difficult to implement. Regional innovation systems, collaborative governance models or the smart specialisation strategies known as RIS3 ${ }^{1}$ are cases in point.

One of the factors that has contributed most to the results obtained in the Gipuzkoa Sarean project in terms of innovation in governance and policy is its continuity throughout several mandates. That continuity has enabled structural changes to be made which are becoming institutionalised at the time of writing this document. This has called for commitment on the part of various holders of political office and technical staff at public administrations. All these elected politicians and technical officers are referred to jointly from now on in this document as policy makers.

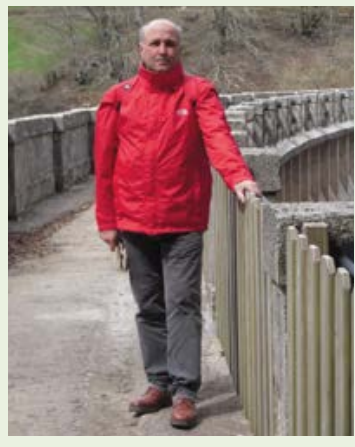

"When the government that set Gipuzkoa Sarean in motion ceased to control the Provincial Council, those of us who were technical officers and academics were left on our own. We all thought that the project that we had in hand was unique and a source of hope. To some extent we felt the type of responsibility experienced by relay runners who must carry the baton onwards so that it does not fall.»

Ander Arzelus, member of the Cabinet Office of the Chief Councillor and a participant in Gipuzkoa Sarean since 2009.

\footnotetext{
${ }^{1}$ For an analysis of the difficulties in implementing RIS3 strategies see Miren Larrea and Miren Estensoro «Overcoming policy making problems in smart specialization strategies: engaging subregional governments». vol. 24 (2016): 1319-1335
} 
The model on which these

guidelines are based was

drawn up jointly by researchers

and policy makers
The core of the guidelines is the Gipuzkoa Sarean model for policy innovation. This model has been developed through action research. Its methodology involves ongoing dialogue between researchers and policy makers, so the model is not a result of academic deliberations among researchers or of practices of policy makers alone. It is a co-generated model, i.e. the result of combining the different types of knowledge brought to the project by researchers and policy makers.

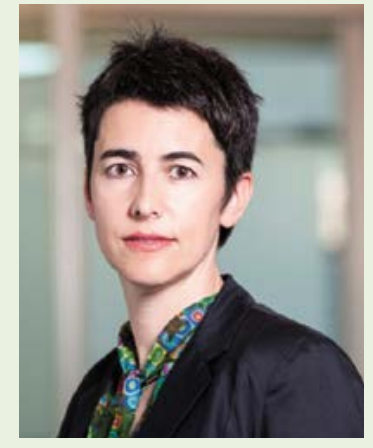

"In 2008 Orkestra made a firm move in the direction of developing action research. This approach has given us a very powerful tool for working with policy makers not «for» practice but «in» practice. Gipuzkoa Sarean has proved itself to be a highly important laboratory for developing our own approach to action research.»

Miren Larrea, researcher at Orkestra, a participant in Gipuzkoa Sarean since 2009 and its academic coordinator since 2010.

\section{Change manifests itself through the behaviour of actors, who gradually form habits that in the long term become a new culture}

Gipuzkoa Sarean is an action research process aimed at creating and consolidating a model of governance based on participation. The goal is to achieve greater efficiency in policy making and to help build a more democratic society. These goals, and efforts to achieve them through the research process, have been shared with an international research team which has validated the process. 


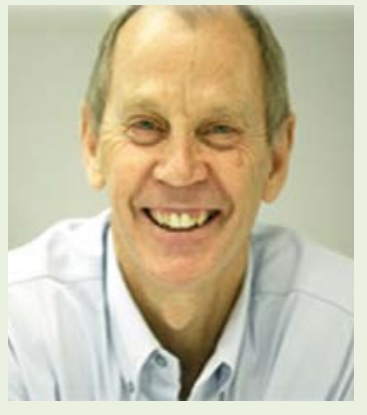

"What you are triying to do here is admirable and as far as I know it is unique. I have lived and travelled a lot in diferent places, and I have never seen a government that has adopted action research as a transformational methodology. I really hope that this process succeeds not just for the success of the Basque Country but also to provide an example to the rest of the world».

Ken Dovey, University of Technology Sydney, Australia.

The Gipuzkoa Sarean project has focused on policy innovation, stressing the need for a previous construction of a new form of governance that facilitates the participation of actors in the relevant territory. These guidelines are structured around the four stages used by this methodology to foster innovation in such processes.

The consolidation of this new form of governance is the main impact of the action research process for policy innovation, over and above one-off milestones, partial consensuses and temporary events. It is an impact visible only in the longterm, but which nevertheless needs to be legitimised by other, short-term impacts. In the Gipuzkoa Sarean project these short-term impacts can be seen in the decrees listed below, which have influenced territorial development policies in Gipuzkoa:

- a decree to foster local job promotion programmes;

- a decree to encourage the analysis of the value chain for energy, in a new financing framework for micro-companies that invest in energy efficiency;

- an internationalisation agreement between the Provincial Council of Gipuzkoa, the Chamber of Commerce of Gipuzkoa and Garapen;

- the organising of events in each county to discuss socio-economic development and build bridges with other actors there. 


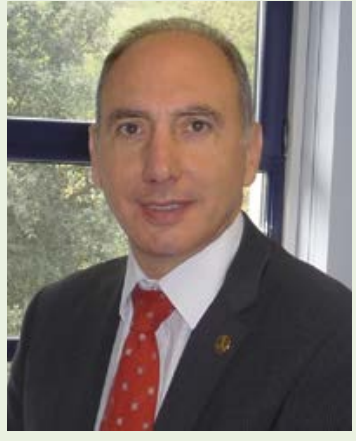

"Regarding energy, the key lies in orienting actions in the county, criteria and experiences to be shared, and in highlighting the importance of educating about energy (both in terms of generation and energy efficiency). Participation is important, and there are many examples of how to extend $i t$; there are also numerous experiences as regards forms of participation».

Juan Angel Balbas, Head of the Debabarrena County Agency.

\section{Rationale and Context}

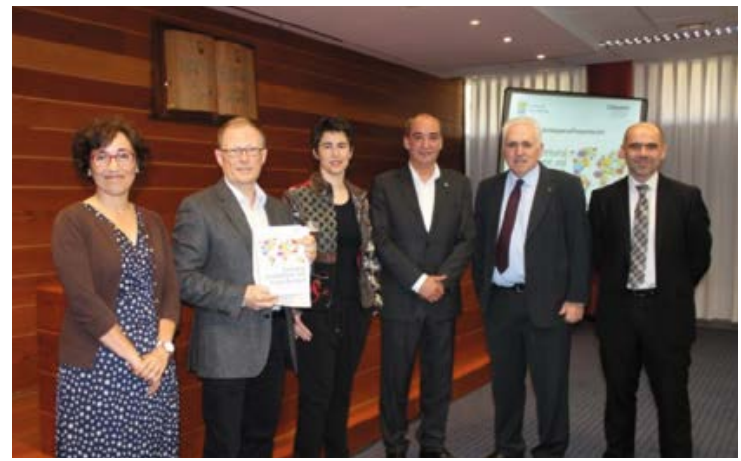

In the period 2009-2016 there has been significant learning on the part of both policy makers and researchers taking part in Gipuzkoa Sarean, regarding how to tackle innovation in territorial development policies, as well as on how to open up such policies to participation by local actors. Part of this learning process has been shared with the academic community through publications made by researchers (see Annex 1).

However, attempts to reach out to policy makers who did not take part in the process have been few in number and poorly focused. An effort was made to create an informative publication that brings together the reflections of the actors that had participated in the process so far $^{2}$. A second example is the document prepared for the RRI-Tools platform, which presents cases of responsible research and innovation practices in Europe ${ }^{3}$.

This briefing, which is intended to provide guidelines for policy makers who face the challenge of innovation in their policies and integrating local actors into them, is therefore seen as significant in filling a gap. It sets out to share the knowledge developed in a way that is useful in the day-to-day work of policy makers at local (municipal councils), territorial (provincial councils) and Basque Government

\footnotetext{
2 Gipuzkoa Sarean. Bidean. Gipuzkoako Foru Aldundia: Donostia - San Sebastián, 2015.

3 Building Bridges: A four step framework to improve territorial governance
} 
(autonomous region) levels. It is based on material that has been systematised over the past years for the project, and therefore includes both the theoretical model and the experiences of the participants in the process.

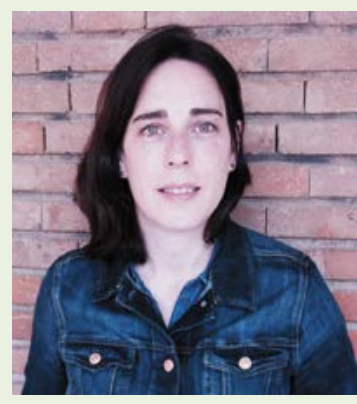

"The whole Gipuzkoa Sarean process is documented. According to Guba E Lincoln, one way in which those who have not taken part in a process can learn from it is to provide them with indirect experience, i.e. to share with them not only what actually happened but also the reasons and motivations underlying it.»

Ainhoa Arrona, Orkestra, member of the Gipuzkoa Sarean research team from 2009 to 2015.

\section{Guidelines for innovation in territorial development policies}

As stated in the Introduction, the Gipuzkoa Sarean process can be summed up in a working model that is co-generated, i.e. that stems from a combination of different forms of knowledge (academic and practical) and also from reflection and action. The various stages of the model are shown in Figure 1.

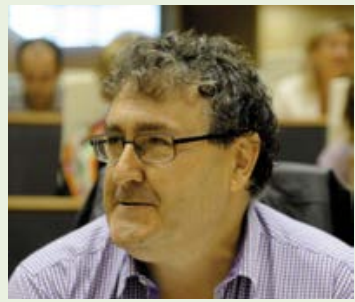

"The researchers have carried out a process of gentle but continuous resistance. The process is cyclical - reflection-policy, reflection-policy - in which the circle is not static but rather moves forward like a wheel».

Juan Karlos Alduntzin, Head of the Cabinet Office at the Provincial Council of Gipuzkoa from 2013 to 2015. 


\section{FIGURE 1. Stages of the policy innovation process}

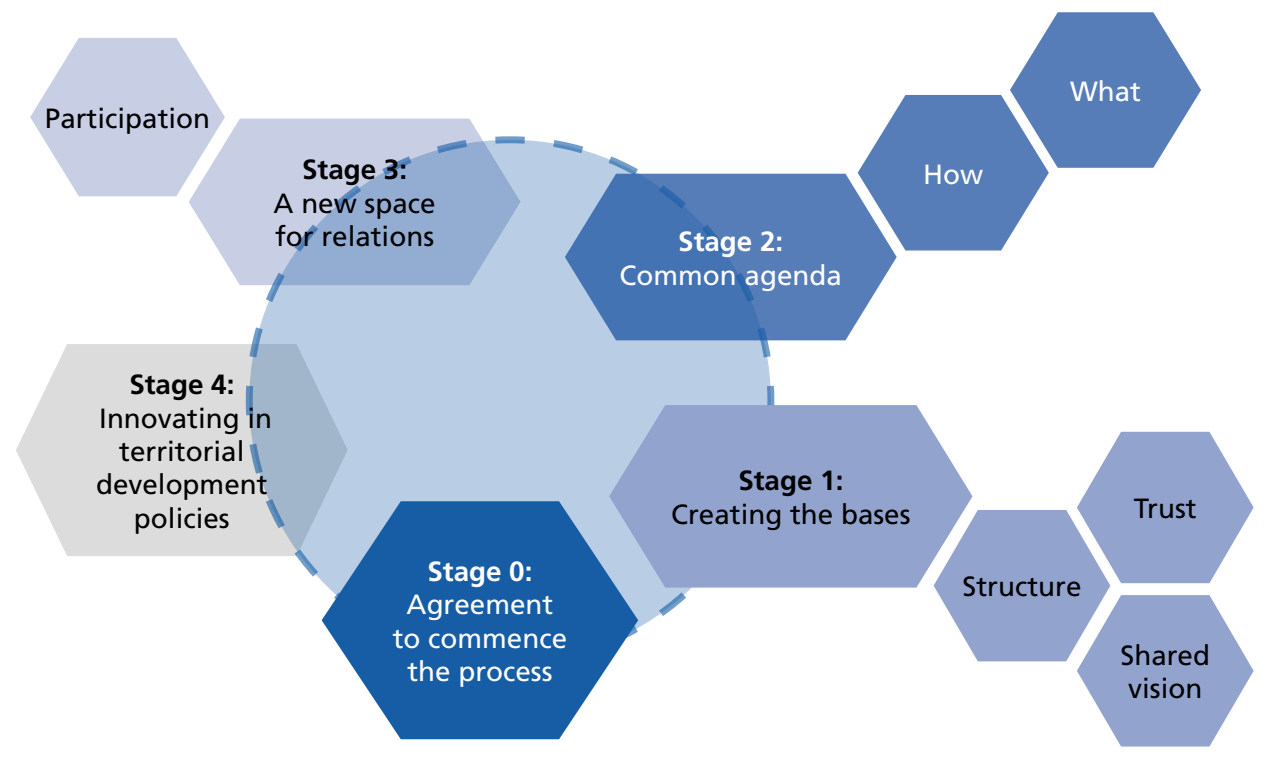

Figure 1 shows the stages required to develop a policy innovation process. However, they must not be seen as linear but rather as a cyclical process in which the later stages feed back into the earlier ones and in which stages sometimes overlap. The sections below describe each of these stages.

\section{STAGE 0: Agreement to commence the process: the challenge of finding a model for collaboration between policy makers and researchers}

The most important point in this stage is for negotiations to be held and agreements reached between policy makers and researchers as to the nature of the problem to be solved (what) and the method to be used to solve it (how).

To negotiate the "how» researchers should put forward an analytical framework for the process. Figure 2 (Karlsen \& Larrea, 2014) shows the framework that represents the process in action research for territorial development. 


\section{FIGURE 2. Creation of collective knowing in the agora}

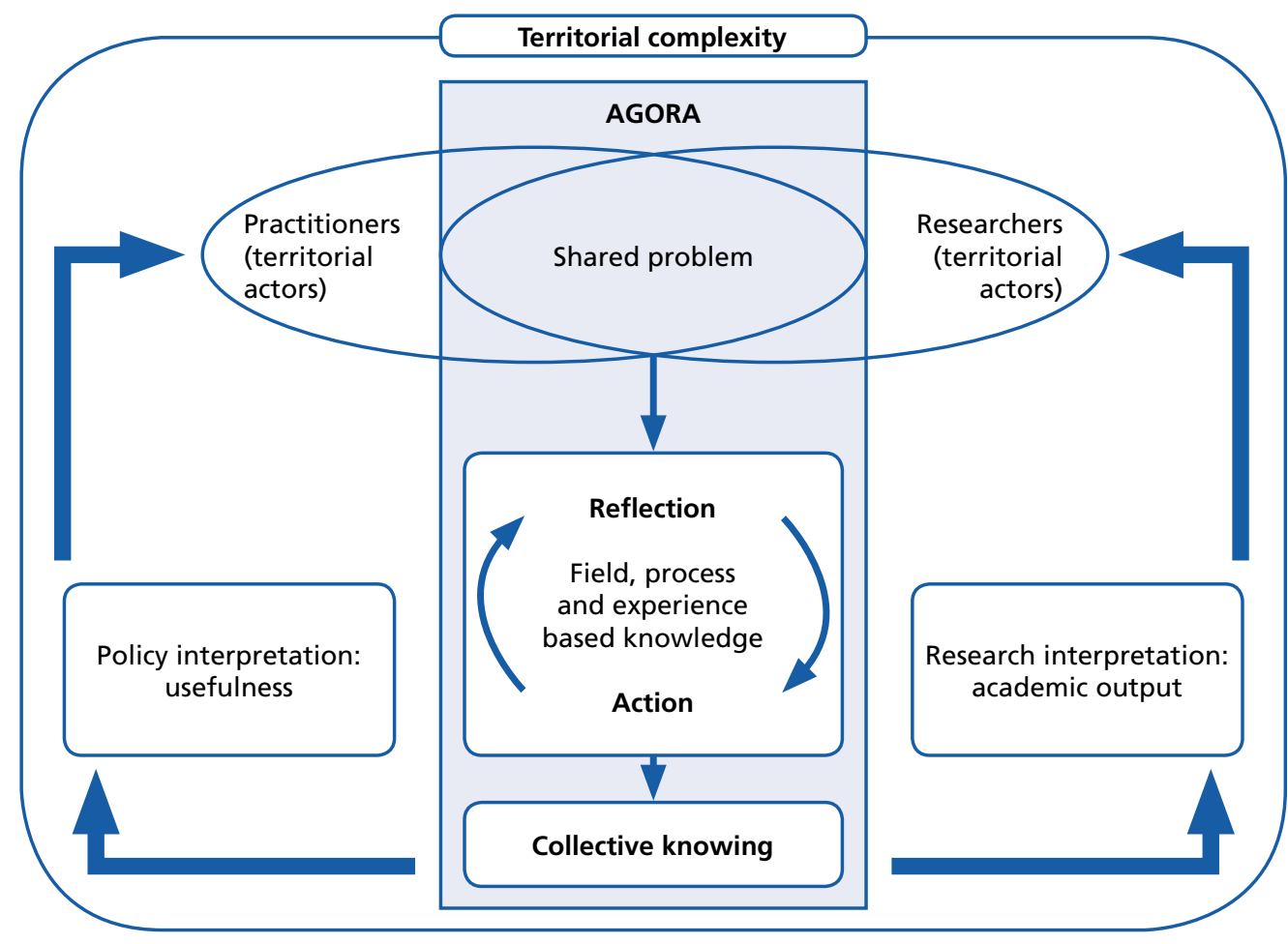

The first point on which policy makers and researchers must reach joint awareness is that the process is to take place in a context of territorial complexity. This means that there will be a variety of actors with potentially different interpretations of where the main problems to be solved by policies lie and what potential solutions exist. There is no single actor in the territory with a hierarchical position that would enable them to impose solutions (though this does not mean that some actors do not have more power than others), so solutions cannot be formulated on a command and control basis. This is why solutions based on dialogue must necessarily be found.

The model also highlights the importance of both policy makers and researchers defining the Agora at the outset as a meeting place for them; a place where politics meets academia, and where policy approaches are strengthened by passing through the filter of academic reflection, just as academic knowledge is subjected to the need to provide value in terms of policy. 


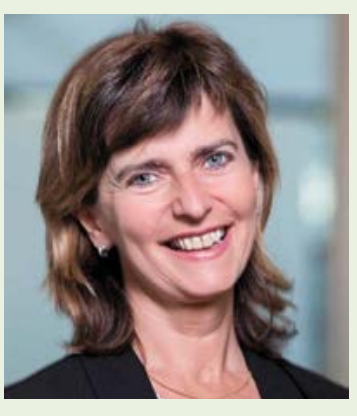

"The part of the process that I value most highly is that both policy makers and researchers have shown themselves to be open to cooperation and to experimenting with new forms of doing research. As a rule, policy makers commission a number of deliverables from a research team which submits a document to them after a few months, but this project was based on dialogue».

Mari Jose Aranguren, Director-General of Orkestra.

As mentioned, in this initial stage it is important to reach a consensus not only regarding the model for the process but also regarding the problem to be solved. This is shown in the model as the "shared problem", and must be understood as the outcome of a process of negotiation in which policy makers and researchers may have different interests. It must be understood that this is not a question solely of a research process aimed at generating new knowledge, nor indeed solely a policy formulation process aimed at solving a problem. It is both at the same time, and the right balance must be agreed from the outset.

Having defined the shared problem, the model proposes the commencement of processes of reflection/action in which policy makers table the problems that they encounter in their process and researchers provide frameworks and concepts to help look at the problem from angles that enable solutions to be drawn up. Policy decisions are always the responsibility of policy makers, and the process advances to the extent that they act on small-scale decisions. This entails redefining problems already posed and defining new problems once the existing ones are solved.

Maintaining these processes over time leads to the production of collective knowledge. Collective knowledge translates into capabilities, and learning in terms of collective action, under which the actors in an agora systematically adapt their actions over the course of time via a learning process. The ability that results from such knowledge can only be developed over time through a learning process that takes place when a group of local actors meet regularly and members interact with one another. This is fostered through dialogue. Dialogue gives rise to connections between theoretical concepts, discourses and actual situations that establish the basis for action and for changing given situations in a territory. In these spaces for dialogue theoretical concepts are discussed and turned into specific actions. Dialogue is a method for inducing change in language, behaviour and organisational and institutional structures. 
What this model cannot convey to others is the required level of commitment. The best way of illustrating this is through the words of the policy makers who participated in the process.

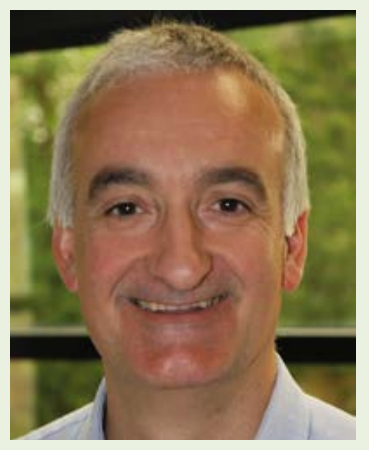

«I am aware that processes shared by policy makers and researchers can fail. I believe that if such processes are to be preserved and are to achieve the results hoped for. then four conditions must be met. The first is political assurances in the sense that the outcome of the process will be upheld. The second is the participation of those who are able to decide and those who hold the relevant knowledge. This is a matter of power, because decision-makers hand over part of their power to a given process. The third is that participants, be they policy makers or researchers, must set aside personal ambitions and prioritise the goals of the project. The fourth and final condition is that policy makers and researchers must feel personal admiration for one another: this is something that goes beyond mere good manners».

Xabier Barandiaran, Head of the Cabinet Office at the Provincial Council of Gipuzkoa and Head of Gipuzkoa Sarean from 2009 to 2011.

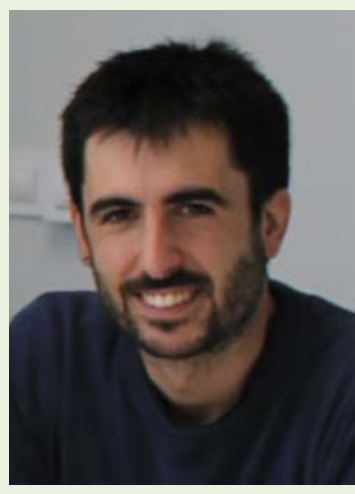

«The participation of researchers has been entirely positive, but has called for constant commitment. Research requires reflection and to reflect one needs time. The method employed called for weekly meetings, and this was a major effort. Effort and commitment, respect for the methods used and loyalty to the process are required. Maintaining such a commitment is not easy.»

Ugaitz Iturbe, member of the Directorate for Territorial Development at the Provincial Council of Gipuzkoa from 2013 to 2015.

The way in which the problem is defined is critical in creating collective knowing. However fragile it may be, this initial consensus is essential for tackling subsequent stages. Consensuses give the process credibility because they are the result 
of decisions and actions. The way in which actors approach the problem determines their involvement and their potential for helping to solve it.

\section{STAGE 1: Creating the basic conditions for the process of policy innovation}

How can the bases necessary to guarantee the consistency of the process be created? In what aspects do policy makers and researchers need to intervene to consolidate those bases? The main argument is that collective knowledge is the result of building social capital in its different dimensions. Such knowledge does not stem from a linear transfer of knowledge from those who know the answer to those who have a problem. In other words this is not a model in which researchers first find solutions to the problems of policy makers and then make recommendations, but rather a process in which they work together to generate solutions. One of the reasons why processes stagnate is that attempts may be made to move forward without first establishing basic conditions. There are three basic conditions for tackling a process of this kind: the creation of spaces for dialogue, the building up of trust-based relationships and the construction of a shared vision of the project.

\section{a. Creating spaces for dialogue}

Dialogue establishes connections between theoretical concepts, discourses and actual situations which provide the basis for action and for changing a given situation in a territory. In spaces for dialogue theoretical concepts are discussed and turned into specific actions. Dialogue is a way of introducing changes in language, in behaviour and in organisational and institutional structures. The following questions may help to create spaces for dialogue.

- What is the status of each participant (the role that they play), and therefore what is their decision-making capacity?

- Have all participants taken on board their status?

- Is the status of each participant acknowledged by everyone?

\section{b. Building up trust-based relationships}

Trust is the best catalyst for dialogue. Dialogue is based on the trust that the parties in the discussion have in one another and the extent to which they believe that each has something valuable to contribute to the other. Trust is built up when discourse is consistent with practice, when people are seen to be listening 
sincerely, whether or not they agree with what is said. Dialogue is based on interdependence and on acknowledgement of the other party.

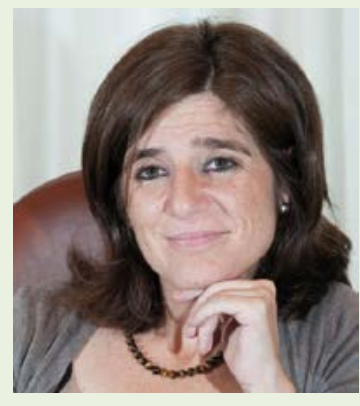

«Participation and trust are basic aspects. Among local actors, especially political actors, there is a great lack of trust. Moreover, our electoral system highlights differences. We should work on models for participation and on sharing projects. Work in this field is necessary if we are to be able to undertake any other projects.»

Helena Franco, Provincial Minister for the Treasury at the Provincial Council of Gipuzkoa from 2011 to 2015.

Trust is not automatic: it must be gained. It cannot be required or imposed. Spaces for innovation in policy therefore require preliminary work: a sort of «slow cooking" process in which trust is built up.

A strategy frequently used to build up trust is to move quickly from discourse to small-scale, local actions which can serve much better than words as a building block. A small action carried out successfully generates trust because it enables slightly more ambitious actions to be undertaken in the next round. On other occasions there is a need to set up transparent forums for information flows, or to work to ensure that agreements are fulfilled.

The following questions may help in considering whether a space based on trust has been established.

- Do participants trust in the good intentions of everyone else?

- Do participants trust in the capabilities of everyone else?

- Is the process transparent?

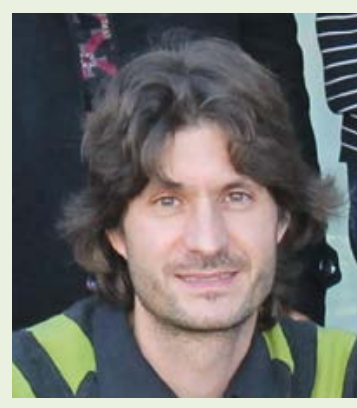

"In Gipuzkoa Sarean we have been able to see the importance of relationships in projects and initiatives, and the way in which trust levels influence outcomes and the paths that processes take. The flexibility of this process has enabled us to make corrections and improvements while it was ongoing.»

Xabier Sanchez, Oarsoaldea County Development Agency. 


\section{c. Constructing a shared vision}

Spaces for interaction stem from a complex need that requires a joint response. The complexity of the problems tackled at territorial level means that they cannot be solved from a short-term project mindset: there is a need for a long-term process outlook to generate evolutionary change. This means that when working with policy innovation processes short-term expectations must be dealt with. This in turn calls for a critical examination of the dynamics that have led to the current situation. The purpose of dialogue in this context is to generate short-term decisions aligned with the long-term construction of solutions.

Striving to achieve this balance between the short and long-term outlooks, the shared vision at Gipuzkoa Sarean was not interpreted as meaning that everyone should necessarily think the same thing. The shared vision was defined as a shared interpretation of differences, i.e. an attempt by everyone to build up a narrative concerning the various interpretations held by participants in regard to the problems to be dealt with and their potential solutions. This shared interpretation of differences gave rise to better conditions in which to seek spaces (sometimes niches) for agreement on action.

The following questions may help to determine whether a shared vision has been established:

- Can the participants explain what the process consists of?

- What differences are there in the way in which participants interpret the general goal of the process and the philosophy on which its implementation is based?

- Is there a raft of minimum elements shared by everyone?

\section{STAGE 2: Defining a shared agenda}

Once the basic conditions are established as indicated above, the next step is to draw up a shared agenda to set out clearly what the co-generation process is expected to achieve. However it must also specify how those goals are to be achieved.

The "how» is one of the most neglected areas in policy innovation processes. 


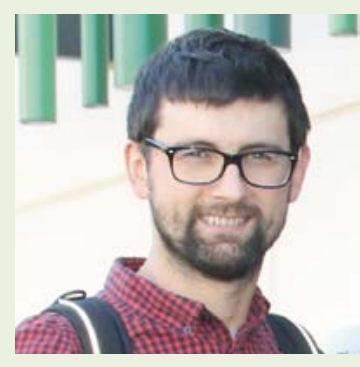

"The most innovative feature of Gipuzkoa Sarean is the reflection concerning the process. In any process there is a "what» and a «how», and I believe that it is essential to give proper importance to the "how». This is what Gipuzkoa Sarean has taught us.»

Borja Urretabizkaia, Facilitator at the Goierri County Development Agency.

Collaboration, which is frequently signalled as a tool for policy innovation, is not an easy process in which everyone works together on the basis of good intentions. It is in fact a process of managing the conflicts that emerge when participants have different interpretations as to what the problems in a territory are and how they can be solved. That is why this section devotes a substantial amount of space to dealing with conflict.

\section{Conflict is necessary to}

generate new contexts that

\section{can permit new interactions}

\section{based on consensus}

Conflict is overcome not by unifying outlooks but by learning to integrate differences into the action taken. This is why the process is not linear. It is hard to determine the outcome of a networked construction process a priori when that process depends on, and requires contributions from its participants.

One of the critical points in fostering territorial development is understanding that it is only natural that there should be conflict, and not something that must be avoided. It is a question of mainstreaming and assessing the relative importance of conflict as an inherent part of the complexity of human relations. This must be accepted as a fact so that new perspectives, knowledge and innovations can be built up. Conflict means both crisis and opportunity.

It should be borne in mind that for conflict to be managed it must be rendered explicit, and that this can only be done at a cost, because it generates tensions and hampers relations. In general, for a local actor to be willing to express conflict explicitly he/she must have some expectation that it may be resolved and that if it is, then the benefits will outweigh the cost. Figure 3 (Karlsen \& Larrea, 2014), presents a framework for reflection that can be useful as a tool for helping to understand situations and seek to resolve them. 


\section{FIGURE 3. Types of territorial complexity}

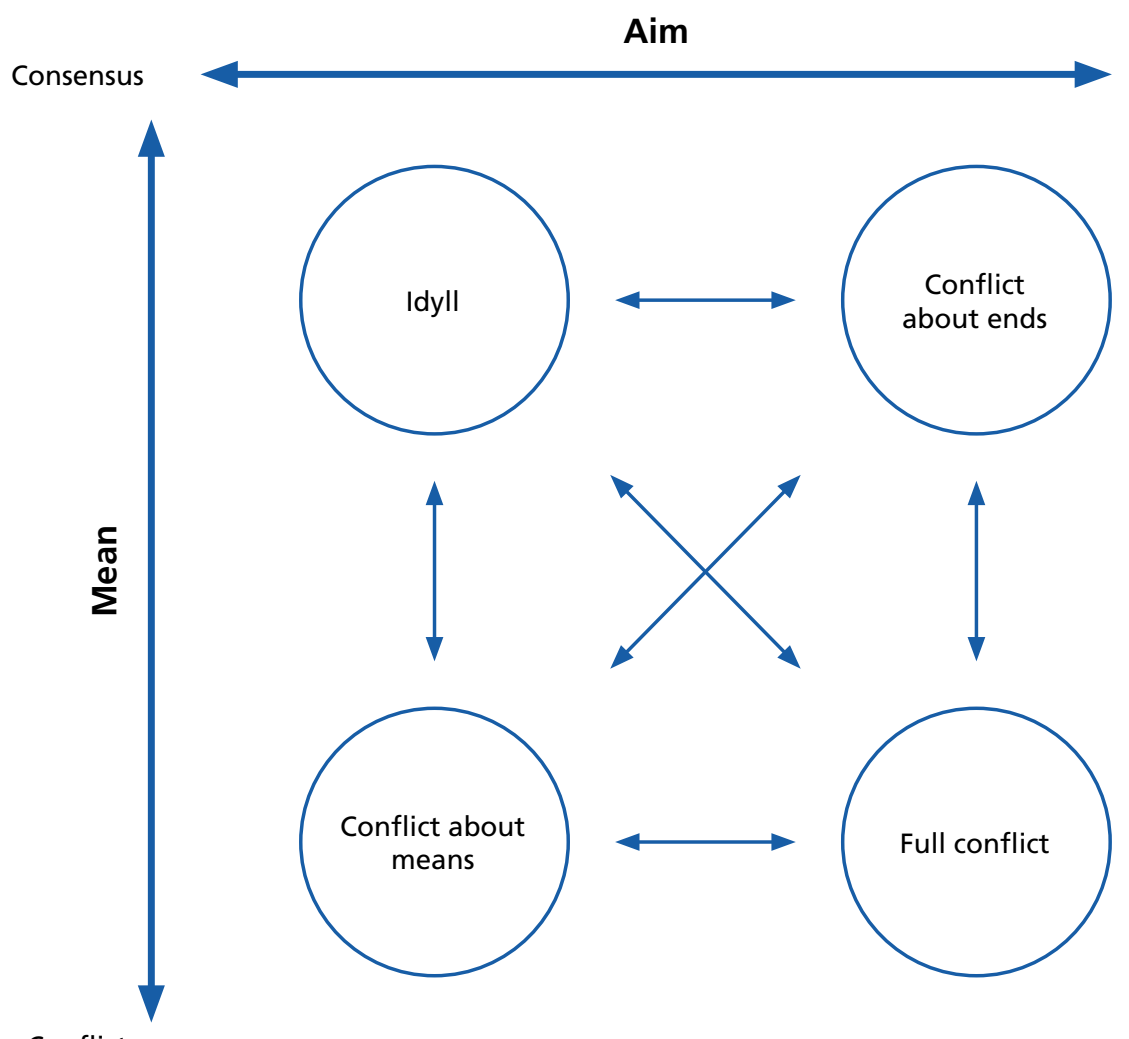

Conflict

In most policy innovation processes that failed to progress as expected there is some kind of conflict concerning the goals of the process or concerning means (financial resources, roles, etc.). Rendering these conflicts explicit and subsequently managing them is a way of carrying the process towards a point of consensus.

When a process reaches a point of consensus, however fragile it may be, a window of opportunity for action opens up that could once again change the balance. With a new balance new conflicts may arise, thus necessitating the continuation of dialogue. This is why one must keep the process viewpoint in mind and see it as a cyclical process in which conditions for acting are generated from time to time.

The five stages described below illustrate the development of conflict in a territorial development policy innovation process. 


\section{a. Generating awareness of conflict}

When there is an apparent consensus in a situation, or a consensus in regard to the main goals, but nothing specific is done there is usually a tacit conflict. One of the tasks of researchers is to help other actors to detect such conflicts.

In a co-generative process policy makers can go to researchers for discussion of what the main tacit conflicts in the process are.

\section{b. Rendering conflicts explicit}

Another task of researchers is to help other actors to render conflicts explicit in a constructive way. There is no sure-fire recipe for solving conflicts, but it is important to design rules in the expectation that different actors will have different viewpoints and interests, and to set up mechanisms through which these differences can be formulated explicitly.

The frameworks and concepts that researchers bring to the process with a view to creating a shared language can help to render conflicts explicit, as they encourage participants to begin to discuss problems on a more theoretical level and then go on to be more specific.

\section{c. Tackling conflict between network and hierarchical structures}

The method proposed here is intended to develop participative policies that include local actors, who normally opt to develop collaboration networks. It is important to remember that although these networks feature horizontal structures that go beyond conventional hierarchical systems there will always be some hierarchical influence on the process. This is because hierarchies within organisations influence the way in which the representatives of those organisations collaborate, and because not all the actors involved have the same status in the process. 
Researchers can play the role of facilitators in the process, to help network relations to bridge the gaps between different cultures of collaboration and differences in status among participants.

\section{d. Keeping dialogue alive}

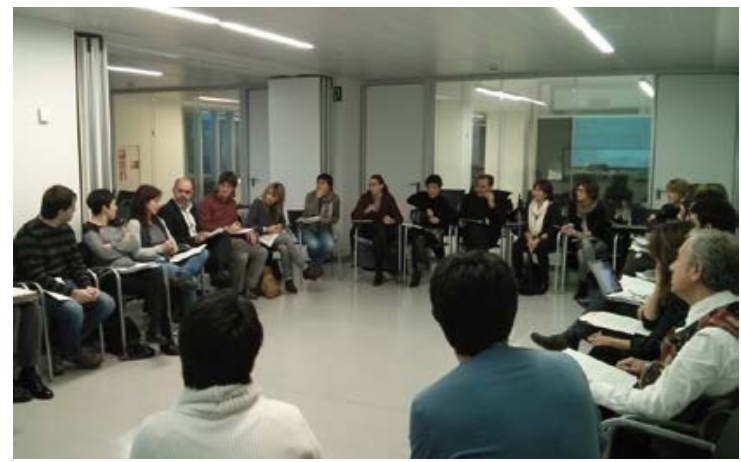

In every process there are moments of crisis in which it is not easy to maintain the dialogue going. The contribution of researchers in such contexts is to create new opportunities for dialogue by submitting concepts and conceptual frameworks that can help to move the process in new directions and prevent it from stagnating. Formal spaces are important, and designing them in such a way as to permit dialogue is a decisive factor. But in a long-term process there will be times when dialogue must also take place in informal settings.

To sustain dialogue researchers must take part not only in the formal spaces but also in the informal settings. Trust in researchers on the part of policy makers is what enables the former to access the latter.

\section{e. Consolidating an action-research strategy for policy innovation}

The researchers' role is not to propose a final, theoretical solution to conflicts but rather to provide participants with concepts and conceptual frameworks that can be used for doing this in practice. Even though the co-generated solution may seem imperfect from a theoretical viewpoint, its outcomes have a greater likelihood of actually being implemented. 


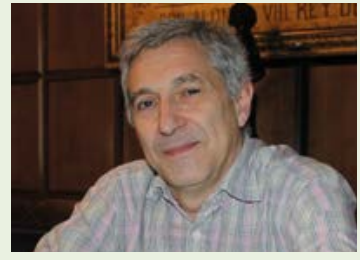

«Researchers do not tell us what we should do: they help us to reflect and it is us who construct our own solutions»..

Sebastián Zurutuza, Head of Management Strategy at the Provincial Council of Gipuzkoa and Head of Gipuzkoa Sarean since 2015.

Action research for policy development requires researchers to take on a role to which they are not always accustomed: they are not only asked to recommend policies but also to accompany policy makers in the search for their own solutions. This change depends not only on researchers but also largely on what is asked of them by policy makers. In other words, implementing this approach depends on developing demand in this sense from policy makers.

\section{STAGE 3: Constructing a new space for relations}

Once a shared agenda has been established as to what is to be done and how, the time comes to take action. This is the point at which many policy innovation processes grind to a halt. It is hard to shift from words to deeds.

Having a shared agenda does not mean having a new, ready-made model of governance that permits policy innovation. It is in actually implementing that agenda that new governance schemes are really built up. At this point the model continues to highlight dialogue as a working tool, but dialogue is much more than just conversation: it must be linked to practical action so as to transfer what is said to a real-world context. Change is the result of processes of dialogue, because through language new interpretations of reality are constructed that can lead to new behaviour patterns and, ultimately, to new practices. Changes in patterns of communication are the spearhead of the process of change.

Changes do not just happen. Whenever one analyses a process

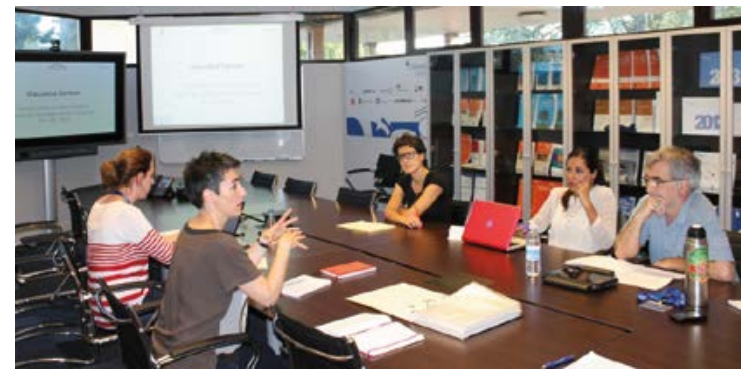


of change, one finds people behind it who made it possible, i.e. people who pushed the process forward.

These people are known as facilitators. Facilitating is a way of working that finds its expression in actions to foster settings for interaction and dialogue. This role is not assigned directly to a specific individual: facilitation actions can be taken by different people at different times.

In territorial development, facilitation is important because such development takes place in a context of conflict. The task of the facilitator is to create the conditions for actors to engage in dialogue and thus reach a consensus and move on to take action. There are times when facilitation requires sound leadership, but the type of leadership provided by the facilitator is not hierarchical but rather relational, based on the recognition of the various actors and built up on the basis of practical work day by day.

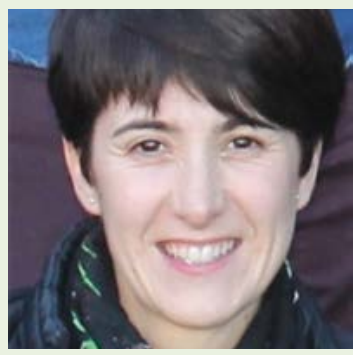

"Initially I saw myself as a technican, but now I have taken on board my role as a facilitator. I know what is being asked of us, and I work from a different viewpoint with regard to processes. We are located in Donostia and through this process we are developing a territorial identity. From that viewpoint we have taken a great leap forward».

Alazne Arocena, Fomento San Sebastián.

\section{STAGE 4: Policy innovation as an outcome of the process}

Policy innovation, which is what this stage covers, is the outcome of prior processes of dialogue in which mutual influence gradually changes attitudes and behaviour patterns, and patterns of action become aligned.

The outcome of co-generation depends on the initial agreement between policy makers and researchers and on how the process is carried forward. As a result, outcomes are different in each case. However, one common feature is that final outcomes are always hard to forecast specifically, because they are constructed as they emerge. In other words, the whole process is oriented towards an outcome which is constructed in a participative fashion and cannot therefore be described beforehand. Another common feature is that this joint construction of outcomes requires changes to be made in relational models and in the governance of policies. 
In the case of Gipuzkoa Sarean policy innovation was framed in terms of the construction of policies in a participatory fashion by the Provincial Council of Gipuzkoa and the agencies involved. The specific outcomes were the decrees mentioned at the beginning of this document. However the new form of governance constructed continues to be a source of further initiatives that enable us to continue innovating.

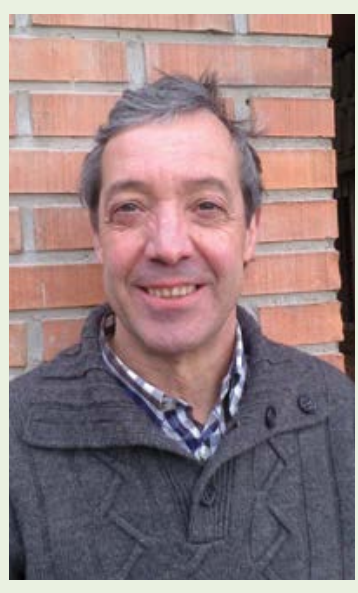

«The county panel [»mesa comarcal»] is a clear example of the new form of governance in my opinion. The intention is to build a new Gipuzkoa by bringing together current circumstances and the challenges facing actors on a county level, from a territorial viewpoint.

When the scheme was set up it was hard to forecast its outcome, because it was an open process so there were no preset paths or results, only a direction. I would say that the most important thing was not the outcome but the process: all that we have learned in the course of that process, relations, different circumstances and priorities at county level, a territorial perspective.»

Jesus Agirre, Head of the Iraurgi Berritzen County Agency.

\section{Conclusions}

Society is experiencing a time of change in which the various tiers of government and universities must adapt and incorporate more participative forms of working that can bring them closer to society. This briefing presents a co-generative form of working that enables this goal to be reached. On the one hand it enables the various tiers of government (and therefore policy makers) to construct spaces for dialogue with other actors in their territories with a view to developing settings for discussion of policies. On the other hand it brings researchers into these processes as facilitators, thus multiplying the social impact of the research carried out.

Establishing the conditions for co-generation is no easy task. It calls for trust building processes and a shared vision, and that means that suitable spaces for dialogue must first be created. This entails investing in a number of intangibles whose outcomes will only become apparent in the long term. In short, such 
approaches involve a calculated risk on the part of both policy makers and researchers.

This briefing does not seek to assess the success of the research project that gave rise to the knowledge shared here. However, we believe that the opinions of the process gathered from policy makers and researchers alike are grounds for considering this as a costly but interesting path for those who seek to reformulate policies through research, and reformulate research through policies.

\section{Bibliography}

Karlsen J. y Miren Larrea. Territorial Development and Action Research: Innovation Through Dialogue. Gower: Surrey, 2014. 


\section{Biographical notes}

\section{Patricia Canto}

Has been part of the Communication team at Orkestra-Basque Institute of Competitiveness's for a decade. Some of her main responsibilities are publishing the activity report distributed to the Institute's main stakeholders every year as well as coordinating the editorial process of The Basque Country Competitiveness Report, the flagship publication that the Institute shares with society every two years. She is currently working on her PhD in which she is exploring the link between communication and the social impact of research. Prior to working at Orkestra she worked at the Ministry of Economy of Mexico. She has taught at the University of Deusto and at the Instituto Tecnológico de Estudios Superiores de Monterrey (Mexico).

\section{Ion Irurzun}

Joined Orkestra-Basque Institute of Competitiveness as a Research Facilitator recently with the aim of further developing the role that facilitators play in research processes. His professional career has a strong orientation towards facilitation in cooperation processes and it is in this area that he has focused over the past years within the project Gipuzkoa Berritzen, whose purpose was the promotion of innovation in cooperation. His experience is essentially practical in forming and developing the network as a cooperation process. He has focused on conflict as a condition for the evolution of such processes.

\section{Miren Larrea}

Is a senior researcher at Orkestra-Basque Institute of Competitiveness. She teaches at the University of Deusto and regularly collaborates with the National Technological University, Rafaela Regional Faculty (Argentina) and with the University of Agder (Norway). Her areas of expertise in research are learning processes in policies, local development, network management and modes of governance that facilitate collaboration. She has practical experience in developing local networks for collaborative learning and has published at the national and international levels in those areas. She currently coordinates a number of projects with municipal and regional governments of the Basque Country that have allowed her to develop an approach to action research for territorial development, one of the building blocks of Orkestra's strategy for transformative research. 


\section{Annex 1. Academic publications arising from the Gipuzkoa Sarean project}

Barandiaran, Xabier y Kepa Korta. Capital social y valores en Gipuzkoa. Diputación Foral de Gipuzkoa. Donostia - San Sebastián: Diputación Foral de Gipuzkoa, 2011.

Costamagna, Pablo. Política y formación en el desarrollo territorial. Bilbao: Publicaciones Deusto, 2015.

Karlsen, James y Miren Larrea. «Moving context from the background to the forefront of policy learning: Reflections on a case in Gipuzkoa, Basque Country» Environment and Planning C: Government and Policy (2016): 1-16. doi: 10.1177/0263774X16642442.

Karlsen J., y Miren Larrea. «Interaction between Research and Practice: Editorial». International Journal of Action Research, 10, n 2 (2014): 125-128.

Karlsen J. y Miren Larrea. Territorial Development and Action Research: Innovation Through Dialogue. Gower: Surrey, 2014.

Karlsen J. y Miren Larrea. «The contribution of Action Research to Policy Learning: The Case of Gipuzkoa Sarean». International Journal of Action Research, 10, n 2 (2014): 129-155.

Larrea, Miren. «¿Hacia dónde evoluciona la participación para el desarrollo local? Reflexiones desde Gipuzkoa». En Ciudadanía Empoderada: cultura y participación para el desarrollo loca, editado por Ricardo Calvo, Juan A. Rodríguez, A., Jaume Portet; Mónica Bou y José M. Romera. Valencia: Germania, s.I., 2013.

Larrea, M., María José Aranguren, y James Karlsen. «The Policy Process in Regional Innovation Systems: Targeting Behavioural Value Added of Policy in Gipuzkoa, Basque Country». En Perspectives on Global and Territorial Change, editado por Philip Cooke y Mario. D. Parrilli. Londres: Palgrave, 2012.

Larrea, M. y Miren Estensoro «Overcoming policy making problems in smart specialization strategies: engaging subregional governments». European Planning Studies, vol. 24 (2016): 1319-1335. 




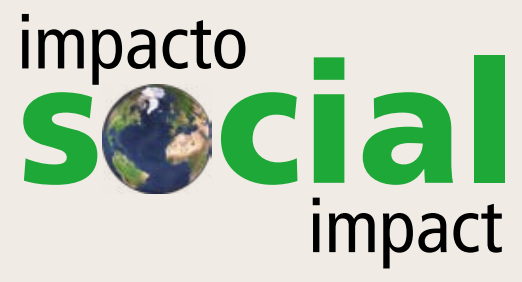

